\title{
O OPERADOR DE WIGNER APLICADO A COLISÕES DE MUDANÇA DE ESTRUTURA FINA ENTRE ÁTOMOS ALCALINOS NO REGIME FRIO
}

\section{Helena Carolina Braga}

Dissertação apresentada ao Instituto de Física de São Carlos, da Universidade de São Paulo, para obtenção do título de mestre em Ciências: "Física Básica".

Orientador: Prof. Dr. Reginaldo de Jesus Napolitano

$0^{k}$ USP/IFSCISBI

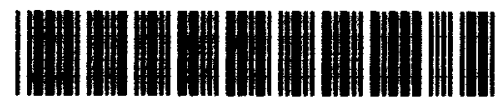

8-2-001443

São Carlos

2002 
Braga, Helena Carolina

"O operador de Wigner aplicado a colisões de mudança de estrutura fina entre átomos alcalinos no regime frio"/ Helena Carolina Braga - São Carlos, 2002

Dissertação (Mestrado) - Instituto de Física de São Carlos da Universidade de São Paulo, 2002 - páginas: 84

Área: Física Básica

Prof. Dr. Reginaldo de Jesus Napolitano

1. Colisões atômicas, 2. Perdas em armadilhas, 3. Estrutura fina, 4. Funçáo de wigner

I. Título 
MEMBROS DA COMISSÃO JULGADORA DA DISSERTAÇÃO DE MESTRADO DE HELENA CAROLINA BRAGA, APRESENTADA AO PROGRAMA DE PÓS-GRADUAÇÃO EM FÍSICA, ÁREA DE CONCENTRAÇÃO FÍSICA BÁSICA, DA UNIVERSIDADE DE SÃO PAULO, EM 22/03/2002.

\section{O M I S S Ã O J U L G A D O R A:}

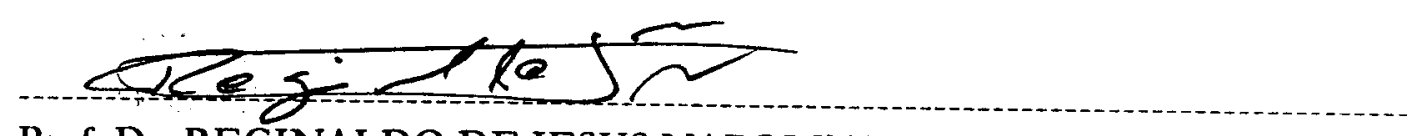

Prof. Dr. REGINALDO DE JESUS NAPOLITANO(Orientador) - IFSC/USP

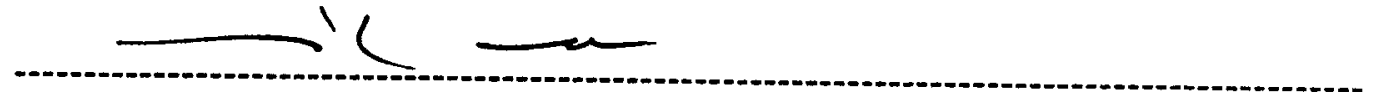

Prof. Dr. MILED HASSAN YOUSSEF MOUSSA - UFSCar

Prof. Dr. ESMERINDO DE SOUSA BERINARDES - IFSC/USP 
Ao meu querido Matheus. 


\section{AGRADECIMENTOS}

- Agradeço primeiramente à FAPESP pelo financiamento deste projeto.

- Ao Prof. Dr. Reginaldo de Jesus Napolitano pela orientação, paciência e amizade.

- Ao Dr. Marcelo Marchiolli pela inestimável ajuda no desenrolar deste trabalho.

- Às secretárias Isabel Rosani e Wla pela atenção e paciência.

- Aos professores e amigos Oswaldo Novaes de Oliveira Jr. (Chu) e Francisco Gontijo Guimarães (Chico) por não me deixarem desistir.

- Aos companheiros Clodoaldo, Yuri, Sílvio e Wander pela acolhida.

- À minha maravilhosa família, a qual eu só aprendi a dar valor com a ausência, em especial à minha mãe pelo exemplo e ao meu pai pelo equilibrio e carinho. Agradeço também, às minhas irmãs Neloca e Paiaia pelo incentivo.

- E por fim, agradeço aos amigos Ronny, Léo-loco, Paulette, Matheus (o outro!), Maurice, Nilvinha, Maira e Claudia. 


\section{Resumo}

Neste trabalho abordamos os processos de perdas colisionais por mudança de estrutura fina e por escape radiativo. Estes processos são importantes por serem os principais fatores limitantes da densidade e da permanência de átomos confinados em armadilhas magneto-ópticas. A utilização do formalismo de Wigner nos possibilitou tratar a dinâmica dos graus de liberdade internos do sistema de forma puramente quântica, enquanto tratamos os graus de liberdade translacionais de maneira semiclássica. Com este formalismo deduzimos equações inéditas para uma colisão unidimensional e desenvolvemos um algoritmo, também inédito, para a resolução numérica de tais equações. 


\section{Abstract}

In this work we study the collision loss processes caused by fine-structure change and radiative escape. These processes are important because they limit the density and the confinement time of atoms in magneto-optical traps. The use of the Wigner-function formalism allows us to treat the internal degrees of freedom purely quantum mechanically, while treating the dynamics of the external degrees of freedom quasi-classically. This dissertation employs this formalism to derive for the first time, quasi-classical equations describing one-dimensional cold collisions, including dissipation due to spontaneous emission, and an algorithm, also for the first time, to solve numerically the mentioned set of coupled equations. 


\section{Sumário}

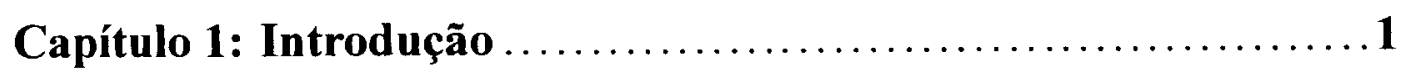

Capítulo 2: Desenvolvimento Teórico $\ldots \ldots \ldots \ldots \ldots \ldots \ldots \ldots \ldots \ldots \ldots \ldots \ldots \ldots \ldots \ldots \ldots$

2.1 O Formalismo de Weyl-Wigner $\ldots \ldots \ldots \ldots \ldots \ldots \ldots \ldots \ldots \ldots \ldots \ldots \ldots \ldots \ldots \ldots \ldots \ldots \ldots$

2.1.1 A transformada de Weyl $\ldots \ldots \ldots \ldots \ldots \ldots \ldots \ldots \ldots \ldots \ldots \ldots \ldots \ldots \ldots \ldots \ldots$

2.1.2 Propriedades matriciais de $\hat{\Delta}(x, p) \ldots \ldots \ldots \ldots \ldots \ldots \ldots$

2.1.3 Traço de operadores a partir de suas respectivas transformadas ...9

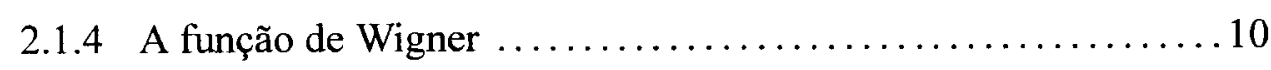

2.1.5 Outras propriedades da função de Wigner $\ldots \ldots \ldots \ldots \ldots \ldots \ldots 12$

2.2 Modelo Simplificado para Três Curvas de Potencial ................ 14

2.3 Evolução da Função de Wigner para o Modelo de Três Curvas de

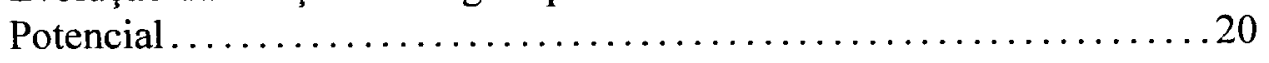

Capítulo 3: Desenvolvimento do Algorítmo $\ldots \ldots \ldots \ldots \ldots \ldots \ldots$

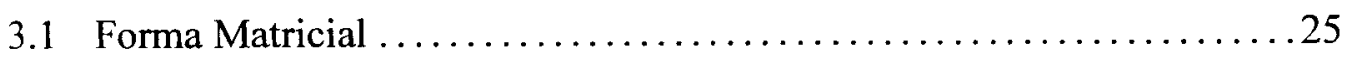

3.2 Resolução das Equações Diferenciais ....................... 27

3.2.1 Operador Partido com Trajetórias $\ldots \ldots \ldots \ldots \ldots \ldots \ldots \ldots . \ldots \ldots$

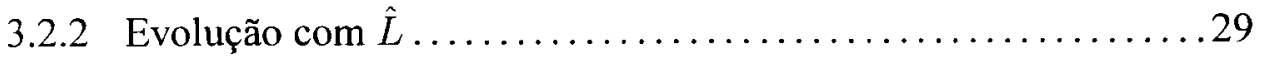

3.2 .3 Evolução com $\hat{M} \ldots \ldots \ldots \ldots \ldots \ldots \ldots \ldots \ldots \ldots \ldots \ldots \ldots \ldots \ldots \ldots \ldots \ldots \ldots$

3.3 Considerações sobre o Algoritmo $\ldots \ldots \ldots \ldots \ldots \ldots \ldots \ldots \ldots \ldots \ldots \ldots \ldots \ldots \ldots \ldots \ldots \ldots$ 
Capítulo 4: Resultados e Discussões ....................... 36

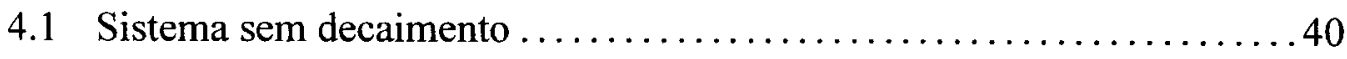

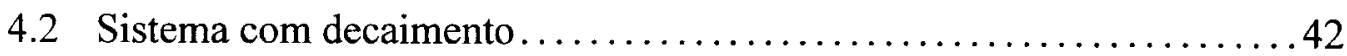

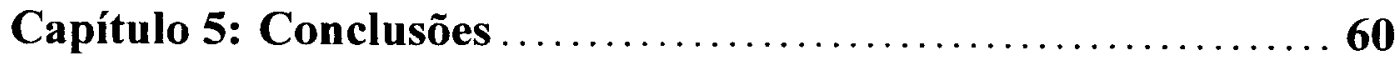

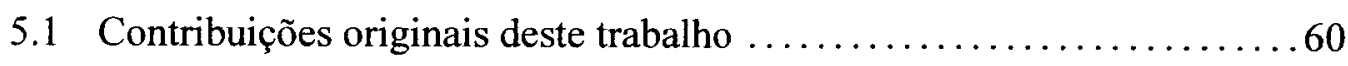

5.2 Conclusões importantes a respeito do modelo $\ldots \ldots \ldots \ldots \ldots \ldots \ldots$

5.3 Sugestões para trabalhos complementares $\ldots \ldots \ldots \ldots \ldots \ldots \ldots \ldots \ldots \ldots \ldots$

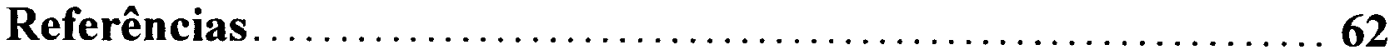

Apêndice A: Cálculo do Hamiltoniano ..................... 63

Apêndice B: Cálculo das Equações Diferenciais ..............6 67

Apêndice C: Soluções Analíticas ....................... 78 


\section{Lista de Figuras}

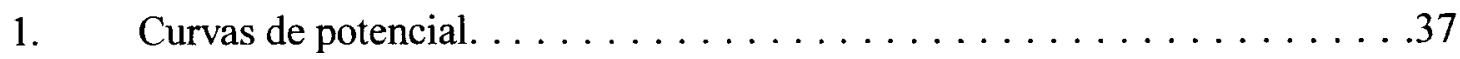

2. Gráfico da distribuição inicial. .......................... 39

3. Gráfico da dinâmica das populações quando não há decaimento. . . . . . 41

4. Função de Wigner no estado excitado após 10ns............. 43

5. Função de Wigner no estado intermediário após $10 \mathrm{~ns} \ldots \ldots \ldots \ldots \ldots 44$

6. Função de Wigner no estado fundamental após $10 n s \ldots \ldots \ldots \ldots \ldots 45$

7. Função de Wigner no estado excitado após $60 n s . \ldots \ldots \ldots \ldots \ldots . \ldots 46$

8. Função de Wigner no estado intermediário após $60 n . . \ldots \ldots \ldots \ldots 47$

9. Função de Wigner no estado fundamental após $60 n s \ldots \ldots \ldots \ldots \ldots 48$

10. Função de Wigner no estado excitado após $200 n s . . . \ldots \ldots \ldots . . .49$

11. Função de Wigner no estado intermediário após $200 \mathrm{~ns} . \ldots \ldots \ldots \ldots 50$

12. Função de Wigner no estado fundamental após $200 n s . \ldots \ldots \ldots \ldots \ldots 51$

13. Gráfico da dinâmica das populações quando há decaimento. ........ 52 
14. Gráfico da distribuição de momentum no estado fundamental antes da colisão.

15. Gráfico da distribuição de momentum no estado fundamental após a colisão. . .55

16. Gráfico da distribuição de momentum no estado intermediário antes da colisão.

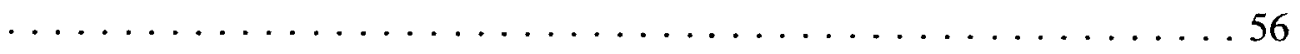

17. Gráfico da distribuição de momentum para o estado intermediário após a colisão. . . . . . . . . . . . . . . . . . . . . . 57

18. Gráfico de população durante a colisão com decaimento 10 vezes maior. . . . . 


\section{Capítulo 1: Introdução}

O principal objetivo deste trabalho foi o estudo de processos colisionais envolvendo átomos frios. O Instituto de Física de São Carlos tem tradição no aprisionamento e resfriamento de átomos através da utilização do laser. Em particular, neste instituto têm-se feito experiências com nuvens de átomos alcalinos confinados por um dispositivo conhecido como armadilha magneto-óptica. Nesta armadilha uma nuvem atômica é aprisionada numa região (em torno da origem de um sistema de coordenadas) em que três pares ortogonais de feixes laser contrapropagantes se cruzam e, onde o campo magnético, devido à presença de bobinas colocadas em configuração anti-Helmholtz, é mínimo. Esta nuvem pode atingir temperaturas de apenas algumas centenas de microkelvins e densidades da ordem de $10^{10}$ átomos por centímetro cúbico. O resfriamento de átomos e suas aplicações renderam a Chu, Cohen-Tannoudji e Phillips o prêmio Nobel de física de 1997[1]

Uma importante motivação para o estudo de processos colisionais entre átomos confinados é que colisões frias mediadas por fótons ressonantes são processos dissipativos. Isto pois o par de átomos colidentes tem tempo bastante para absorver fótons e emiti-los espontaneamente, perdendo, assim, energia. Podemos ilustrar tal afirmação se tomarmos como exemplo o sódio. Átomos de sódio à temperatura típica de $T_{D} \approx 240 \mu K$ (chamada de temperatura de resfriamento Doppler), têm velocidade média de apenas $51 \mathrm{~cm} / \mathrm{s}$, podendo viajar somente $81 \AA$ durante o tempo de vida de seu estado excitado que é de 16 ns. A distância 
interatômica para a transição D2 do sódio é de cerca de $938 \AA$; assim, podemos calcular o tempo de duração de colisões frias, que é da ordem de $(938 \AA ̊)(51 \mathrm{~cm} / \mathrm{s}) \approx 184 \mathrm{~ns}$, um tempo consideravelmente mais longo que o período em que a emissão espontânea ocorre. A repopulação do estado excitado durante a colisão também se torna freqüente para intensidades típicas dos feixes laser em armadilhas magneto-ópticas. Esses processos são, em geral, causadores de perdas. As perdas colisionais de átomos confinados em armadilhas restringem o tempo de confinamento e a densidade de átomos aprisionados. Assim sendo, a dinâmica colisional de átomos frios é um importante objeto de estudo. Estaremos especialmente interessados nos dois principais processos causadores de perdas, que são o escape radiativo e a mudança de estrutura fina, explicados a seguir.

Primeiramente falaremos do escape radiativo. Consideremos um átomo em seu estado excitado de simetria $\mathrm{P}$ acelerado em direção a outro átomo em seu estado fundamental de simetria S. Após ganharem uma certa quantidade de energia cinética relativa, pode haver emissão espontânea pela "quase-molécula". Esta transição radiativa não afeta, essencialmente, o movimento relativo dos átomos e tem como efeito produzir dois átomos em seus estados fundamentais com energia cinética relativa maior do que quando se aproximaram pela primeira vez, cada átomo do par colidente recebe o mesmo acréscimo de energia. Se este acréscimo de energia cinética relativa for maior do que a profundidade em energia potencial da armadilha magneto-óptica, o par de átomos escapa da nuvem aprisionada no processo conhecido como escape radiativo.

A mudança de estrutura fina ocorre da seguinte forma. A emissão espontânea pode deixar de ocorrer. Quando isto acontece para um par atômico em colisão, sob a ação de 
um potencial atrativo, durante um período suficientemente longo, o par atômico pode se aproximar o suficiente para que haja a mudança de estrutura fina. Suponhamos que a "quase-molécula", no início da colisão, corresponda a um átomo excitado no estado de simetria ${ }^{2} P_{3 / 2}$ e a outro no estado fundamental ${ }^{2} S_{1 / 2}$. A curva de potencial atrativa que se correlaciona assintoticamente com este estado cruza, num ponto $R_{c}$, com a curva de potencial repulsivo que se correlaciona assintoticamente ao estado de dois átomos, um no estado excitado ${ }^{2} P_{1 / 2}$ e o outro no fundamental ${ }^{2} S_{1 / 2}$. Assim, existe uma probabilidade não nula de que a "quase-molécula" mude de estado eletrônico em torno de $R_{c}$ e passe a sofrer dissociação por causa da interação antiligante. Ao se separar, os dois átomos ganham energia cinética relativa por causa da aceleração sobre o potencial repulsivo. Cada um dos átomos idênticos recebe o mesmo acréscimo de energia cinética. Este aumento é, em geral, suficiente para que o par atômico escape da nuvem confinada, no fenômeno conhecido como colisão de mudança de estrutura fina. No caso do sódio, por exemplo, o ganho de energia cinética é de cerca de $12 \mathrm{~K}$, enquanto que a armadilha magneto-óptica tem profundidade típica da ordem de $1 \mathrm{~K}$ [2].

Neste trabalho trataremos preferencialmente das colisões de mudança de estrutura fina. Para tanto, utilizaremos um modelo de três curvas de potencial para colisões entre átomos de sódio. Nossos desenvolvimentos serão feitos lançando mão do formalismo de Wigner [3] [4] [5], onde podemos tratar a dinâmica externa do problema de maneira quasiclássica enquanto que os graus de liberdade internos continuam puramente quânticos, o que é uma simplificação importante no tratamento de sistemas onde vários canais participam do espalhamento. Fizemos assim a evolução temporal, na representação de Heisenberg, do 
operador de Wigner segundo procedimento análogo ao descrito por Cook [6] e obtivemos um conjunto de equações diferenciais acopladas como mostrado no Capítulo 2.

Em seguida, no Capítulo 3, fizemos um breve apanhado dos métodos numéricos utilizados para a resolução do sistema de equações acima. O principal deles é conhecido como método do operador partido, uma variante do método de Euler, onde podemos separar as equações diferenciais em duas ou mais equações. Este método foi rebatizado por nós como método do operador partido com trajetórias, uma vez que uma das equações resultantes é uma equação clássica de trajetória, a equação de Liouville. Neste ponto acrescentaremos, também, termos de decaimento com o objetivo de fazer uma abordagem ao escape radiativo.

No Capítulo 4, por sua vez, apresentaremos os resultados obtidos numericamente para resolução das equações diferenciais, mostraremos os gráficos de população de cada um dos três estados e a evolução da função de Wigner durante todo o processo colisional. Este algoritmo nos permite monitorar a dinâmica colisional, o que é inteiramente novo uma vez que os outros formalismos nos fornecem apenas resultados de estados estacionários, com exceção do método de Monte Carlo quântico de pacotes de onda, que demanda recursos computacionais de forma demasiada [7]. 


\section{Capítulo 2: Desenvolvimento Teórico}

\subsection{O Formalismo de Weyl-Wigner}

Desde o advento da mecânica quântica vem se buscando uma maneira de se representar um sistema quântico através de funções de distribuição simples análogas às da mecânica clássica, ou seja, sem a utilização de operadores quânticos, utilizando ao invés disso funções dependentes somente das variáveis de estado $x$ e $p$. Classicamente, um sistema pode ser descrito por uma função de distribuição de probabilidade $P_{c l}(x, p, t)$ dependente somente das variáveis $x$ de coordenada e $p$ de momentum, e do tempo $t$. O valor médio de uma função qualquer $A(x, p)$, associada a um observável físico $A$, neste caso, é dado por

$$
\langle A\rangle_{c l}=\int d x \int d p A(x, p) P_{c l}(x, p, t)
$$

Quanticamente o valor médio, ou esperado, de um operador $\hat{A}(\hat{x}, \hat{p})$ associado a um observável $A$ é dado por

$$
\langle A\rangle_{Q}=\operatorname{Tr}[\hat{\rho}(t) \hat{A}]
$$

sendo que $\hat{\rho}(t)$ é o operador densidade de estado que descreve o sistema quântico em questão e que é definido como

$$
\hat{\rho}(t)=|\psi(t)\rangle\langle\psi(t)|
$$

onde $|\psi(t)\rangle$ é a função de estado do sistema. 
Seria interessante que, dado um sistema quântico, houvesse uma expressão $A(x, p)$ associada ao operador $\hat{A}$ e uma função de distribuição de probabilidade $P_{Q}(x, p, t)$ associado a $\hat{\rho}(t)$ (veja que $A(x, p)$ e $P_{Q}(x, p, t)$ não são operadores, pois dependem somente dos valores clássicos de $x$ e $p$, servindo o índice $Q$ apenas para indicar a procedência da distribuição) tal que

$$
\begin{gathered}
A(x, p) \rightleftharpoons \hat{A}, \\
P_{Q}(x, p, t) \rightleftharpoons \hat{\rho}(t), \\
\langle A\rangle_{Q}=\int d x \int d p A(x, p) P_{Q}(x, p, t) .
\end{gathered}
$$

Devido ao princípio de incerteza de Heisenberg, é impossível determinarmos uma função $P_{Q}(x, p, t)$, pois é impossível determinarmos simultaneamente a posição e o momentum da partícula. Mesmo assim, várias propostas foram feitas para se derterminar funções com propriedades semelhantes às esperadas para $P_{Q}(x, p, t)$. Estas funções são conhecidas como funções "quase-probabilísticas". A primeira delas foi introduzida por Wigner em 1932 [3] com a intenção de estudar correções quânticas para a estatística clássica. A relação entre o operador $\hat{A}(\hat{x}, \hat{p})$ e sua função correspondente $A(x, p)$ é dada pela transformada de Weyl [8], que será estudada adiante.

\subsubsection{A transformada de Weyl}

\section{Princípios}

A descrição de um sistema quântico mapeado no espaço de fase é possível se, e somente se, a cada operador $\hat{A}$ qualquer, existir uma correspondente função $A(x, p)$. 
Tal correspondência é feita via transformada de Weyl.

Dado um operador $\hat{A}$ podemos escrevê-lo como

$$
\hat{A}=\int d x \int d p A(x, p) \hat{\Delta}(x, p)
$$

sendo

$$
A(x, p)=\frac{1}{\pi \hbar} \int d u\langle x+u|\hat{A}| x-u\rangle \exp \left(-\frac{2 i p u}{\hbar}\right)
$$

e

$$
\hat{\Delta}(x, p)=2 \int d u \exp \left(\frac{2 i p u}{\hbar}\right)|x+u\rangle\langle x-u| .
$$

As expressões (2.2) e (2.3) são, respectivamente, a transformada de Weyl e uma base de operadores.

De uma forma totalmente equivalente podemos fazer

$$
\begin{aligned}
& A(x, p)=\frac{1}{\pi \hbar} \int d v\langle p+v|\hat{A}| p-v\rangle \exp \left(\frac{2 i v x}{\hbar}\right) \\
& \hat{\Delta}(x, p)=2 \int d v \exp \left(-\frac{2 i v x}{\hbar}\right)|p+v\rangle\langle p-v| .
\end{aligned}
$$

A base de operadores (2.3) ainda pode ser escrita como

$$
\hat{\Delta}(x, p)=2 \pi \hbar \exp \left[\left(\frac{\hbar}{2 i}\right) \frac{\partial^{2}}{\partial x \partial p}\right] \delta(x-\hat{x}) \delta(p-\hat{p}) .
$$

Esta forma, substituída em (2.1) nos dá

$$
\hat{A}=2 \pi \hbar \int d x \int d p \delta(x-\hat{x}) \delta(p-\hat{p})\left\{\exp \left[\left(\frac{\hbar}{2 i}\right) \frac{\partial^{2}}{\partial x \partial p}\right] A(x, p)\right\}
$$


Esta expressão mostra que, para encontrarmos o operador $\hat{A}$ a partir de sua transformada de Weyl $A(x, p)$, devemos inicialmente calcular $\exp \left[\left(\frac{\hbar}{2 i}\right) \frac{\partial^{2}}{\partial x \partial p}\right] A(x, p)$ e depois substituir as variáveis $x$ e $p$ pelos operadores $\hat{x}$ e $\hat{p}$, respectivamente com a ressalva de se escrever os operadores de coordenada à esquerda dos operadores de momentum.

\subsubsection{Propriedades matriciais de $\hat{\Delta}(x, p)$}

Através da expressão para o operador $\hat{\Delta}(x, p)$ podemos calcular seus elementos matriciais i)

$$
\left\langle x_{1}|\hat{\Delta}(x, p)| x_{2}\right\rangle=\delta\left(x-\left[\frac{x_{1}+x_{2}}{2}\right]\right) \exp \left[\frac{i p\left(x_{1}-x_{2}\right)}{\hbar}\right]
$$

Caso particular

$$
\left\langle x_{1}|\hat{\Delta}(x, p)| x_{1}\right\rangle=\delta\left(x-x_{1}\right)
$$

ii)

$$
\left\langle p_{1}|\hat{\Delta}(x, p)| p_{2}\right\rangle=\delta\left(p-\left[\frac{p_{1}+p_{2}}{2}\right]\right) \exp \left[-\frac{i\left(p_{1}-p_{2}\right) x}{\hbar}\right]
$$

Caso particular

$$
\left\langle p_{1}|\hat{\Delta}(x, p)| p_{1}\right\rangle=\delta\left(p-p_{1}\right)
$$

iii)

$$
\left\langle x_{1}|\hat{\Delta}(x, p)| p_{1}\right\rangle=\sqrt{2 \pi \hbar} \exp \left(\frac{i x_{1} p_{1}}{\hbar}\right) \exp \left[\frac{\hbar}{2 i} \frac{\partial^{2}}{\partial x \partial p}\right] \delta\left(x-x_{1}\right) \delta\left(p-p_{1}\right)
$$

iv)

$$
\frac{1}{2 \pi \hbar} \int d p \hat{\Delta}(x, p)=|x\rangle\langle x|
$$




$$
\frac{1}{2 \pi \hbar} \int d x \hat{\Delta}(x, p)=|p\rangle\langle p|
$$

Destas definições temos

$$
\begin{gathered}
\operatorname{Tr}[\hat{\Delta}(x, p)]=1, \\
\operatorname{Tr}\left[\hat{\Delta}\left(x_{1}, p_{1}\right) \hat{\Delta}\left(x_{2}, p_{2}\right)\right]=2 \pi \hbar \delta\left(x_{1}-x_{2}\right) \delta\left(p_{1}-p_{2}\right), \\
\operatorname{Tr}\left[\hat{\Delta}\left(x_{1}, p_{1}\right) \hat{\Delta}\left(x_{2}, p_{2}\right) \hat{\Delta}\left(x_{3}, p_{3}\right)\right]= \\
=4 \exp \left\{\frac{2 i}{\hbar}\left[\left(p_{2}-p_{3}\right) x_{1}+\left(p_{3}-p_{1}\right) x_{2}+\left(p_{1}-p_{2}\right) x_{3}\right]\right\} .
\end{gathered}
$$

Tais equações serão úties na obtensão do traço de operadores a partir de suas tranformadas de Weyl.

\subsubsection{Traço de operadores a partir de suas respectivas transformadas}

Dados operadores $\hat{A}$ e $\hat{B}$, cujas transformadas de Weyl são, respectivamente, $A(x, p)$ e $B(x, p)$, temos, conforme os elementos de matriz de $\hat{\Delta}(x, p)$, que

$$
\operatorname{Tr}[\hat{A}]=\int d x \int d p A(x, p) \operatorname{Tr}[\hat{\Delta}(x, p)]=\int d x \int d p A(x, p)
$$

Esta propriedade do traço é obtida ao substituirmos (2.10) na equação acima.

Através de (2.11) podemos encontrar o traço do produto dos operadores $\hat{A}$ e $\hat{B}$ :

$$
\begin{aligned}
\operatorname{Tr}[\hat{A} \hat{B}] & =\int d x \int d p \int d x_{1} \int d p_{1} A(x, p) B\left(x_{1}, p_{1}\right) \operatorname{Tr}\left[\hat{\Delta}(x, p) \hat{\Delta}\left(x_{1}, p_{1}\right)\right]= \\
& =\int d x \int d p A(x, p) B(x, p) .
\end{aligned}
$$

É particularmente interessante para nós calcularmos o traço do produto $\hat{A} \hat{\Delta}(x, p)$ : 


$$
\begin{gathered}
\operatorname{Tr}[\hat{A} \hat{\Delta}(x, p)]=\int d x_{1} \int d p_{1} A\left(x_{1}, p_{1}\right) \operatorname{Tr}\left[\hat{\Delta}\left(x_{1}, p_{1}\right) \hat{\Delta}(x, p)\right]=2 \pi \hbar A(x, p) \Rightarrow \\
\Rightarrow A(x, p)=\frac{1}{2 \pi \hbar} \operatorname{Tr}[\hat{A} \hat{\Delta}(x, p)]
\end{gathered}
$$

Este resultado, aliado à expressão (2.12), nos possibilita encontrar uma forma simples para $[\hat{A}, \hat{B}]$ e $\{\hat{A}, \hat{B}\}$, que ficam como

$$
\begin{aligned}
& {[\hat{A}, \hat{B}]=4 i \pi \hbar \int d x \int d p\left\{\sin \left[\frac{\hbar}{2}\left(\frac{\partial^{A}}{\partial x} \frac{\partial^{B}}{\partial p}-\frac{\partial^{A}}{\partial p} \frac{\partial^{B}}{\partial x}\right)\right]\right\} A(x, p) B(x, p) \hat{\Delta}(x, p)} \\
& \{\hat{A}, \hat{B}\}=4 \pi \hbar \int d x \int d p\left\{\cos \left[\frac{\hbar}{2}\left(\frac{\partial^{A}}{\partial x} \frac{\partial^{B}}{\partial p}-\frac{\partial^{A}}{\partial p} \frac{\partial^{B}}{\partial x}\right)\right]\right\} A(x, p) B(x, p) \hat{\Delta}(x, p)
\end{aligned}
$$

Os índices nos operadores (ex.: $\frac{\partial^{A}}{\partial x}$ ) indicam em qual das funções estes atuam.

\subsubsection{A função de Wigner}

A função de Wigner nos é dada quando tomamos a tranformada de Weyl do operador densidade. Através de (2.2) ou (2.4) temos

$$
\begin{aligned}
W(x, p, t) & =\frac{1}{\pi \hbar} \int d u\langle x+u|\hat{\rho}(t)| x-u\rangle \exp \left(-\frac{2 i p u}{\hbar}\right) \\
& =\frac{1}{\pi \hbar} \int d v\langle p+v|\hat{\rho}(t)| p-v\rangle \exp \left(\frac{2 i v x}{\hbar}\right)
\end{aligned}
$$

Ou ainda, por (2.15), 


$$
W(x, p, t)=\frac{1}{2 \pi \hbar} \operatorname{Tr}[\hat{\rho}(t) \hat{\Delta}(x, p)]
$$

Das formas (2.18) e (2.19) temos (estas formas foram escolhidas por ser mais fácil observar as propriedades a seguir, porém todas são equivalentes)

$$
\begin{aligned}
& \int d p W(x, p, t)=|\psi(x, t)|^{2}, \\
& \int d x W(x, p, t)=|\phi(p, t)|^{2},
\end{aligned}
$$

sendo $\psi(x, t)=\langle x \mid \psi(t)\rangle$ (função de amplitude de probabilidade na coordenada de posição) e $\phi(p, t)=\langle p \mid \phi(t)\rangle$ (função de amplitude de probabilidade na coordenada de momentum).

Integrando a função de Wigner nas duas variáveis, temos

$$
\int d x \int d p W(x, p, t)=\int d x|\psi(x, t)|^{2}=\int d p|\phi(p, t)|^{2}
$$

Para obtermos o valor médio de um operador $\hat{A}$ num sistema quântico descrito pela função de estado $|\psi(t)\rangle$

$$
\langle\hat{A}\rangle=\langle\psi(t)|\hat{A}| \psi(t)\rangle=\operatorname{Tr}[\hat{\rho}(t) \hat{A}]
$$

Pelo formalismo da transformada de Weyl temos, segundo (2.14), que o valor médio de $\hat{A}$ pode ser obtido fazendo

$$
\langle\hat{A}\rangle=\int d x \int d p A(x, p) W(x, p, t)
$$

sendo $A(x, p)$ a transformada de Weyl do operador $\hat{A}$. 
Pela definição (2.20), utilizando a forma do operador $\hat{\Delta}(x, p)$ apresentada em (2.5) e tomando o traço no segundo o espaço dos momenta

$$
\begin{gathered}
W(x, p, t)=\frac{1}{2 \pi \hbar} \int d p_{1}\left\langle p_{1}|\hat{\rho}(t) \hat{\Delta}(x, p)| p_{1}\right\rangle \Rightarrow \\
\Rightarrow W(x, p, t)=\frac{1}{\sqrt{2 \pi \hbar}} \exp \left[\left(\frac{\hbar}{2 i}\right) \frac{\partial^{2}}{\partial x \partial p}\right] \exp \left(\frac{i x p}{\hbar}\right) \phi(p, t) \psi^{*}(x, t) .
\end{gathered}
$$

Se, por outro lado, utilizarmos as mesmas expressões, porém, tomarmos o traço segundo o espaço das coordenadas, teremos

$$
\begin{gathered}
W(x, p, t)=\frac{1}{2 \pi \hbar} \int d x_{1}\left\langle x_{1}|\hat{\rho}(t) \hat{\Delta}(x, p)| x_{1}\right\rangle \Rightarrow \\
\Rightarrow W(x, p, t)=\frac{1}{\sqrt{2 \pi \hbar}} \exp \left[\left(-\frac{\hbar}{2 i}\right) \frac{\partial^{2}}{\partial x \partial p}\right] \exp \left(-\frac{i x p}{\hbar}\right) \phi^{*}(p, t) \psi(x, t) .
\end{gathered}
$$

Podemos ver que o lado direito da expressão (2.21) é exatamente o complexo conjugado do lado direito da expressão (2.22). Isto nos leva a concluir que a função de Wigner é sempre real.

Outra característica da função de Wigner está relacionada ao seu módulo. Através de sua definição temos

$$
\begin{gathered}
W(x, p, t)=\frac{1}{\pi \hbar} \int d u\langle x+u|\hat{\rho}(t)| x-u\rangle \exp \left(-\frac{2 i p u}{\hbar}\right) \Rightarrow \\
\Rightarrow W(x, p, t)=\frac{1}{\pi \hbar} \int d u \psi^{*}(x-u, t) \psi(x+u, t) \exp \left(-\frac{2 i p u}{\hbar}\right) \Rightarrow \\
\Rightarrow|W(x, p, t)|^{2} \leqslant\left\{\frac{1}{\pi \hbar} \int d u|\psi(x-u, t)|^{2}\right\}\left\{\frac{1}{\pi \hbar} \int d u|\psi(x+u, t)|^{2}\right\} \Rightarrow \\
\Rightarrow|W(x, p, t)| \leqslant \frac{1}{\pi \hbar} .
\end{gathered}
$$

\subsubsection{Outras propriedades da função de Wigner}

Da definição da função de Wigner dada em (2.18) temos 


$$
W(x, p, t)=\frac{1}{\pi \hbar} \int d u\langle x+u|\hat{\rho}(t)| x-u\rangle \exp \left(-\frac{2 i p u}{\hbar}\right)
$$

Supondo que $|\psi(t)\rangle$ possa ser escrito como uma combinação linear dos auto-estados $|n\rangle$ de um hamiltoniano $\hat{H}$, temos

$$
|\psi(t)\rangle=\sum_{n} a_{n}(t)|n\rangle
$$

o que nos levará a uma expressão de $\hat{\rho}(t)$ da seguinte forma:

$$
\hat{\rho}(t)=\sum_{n} \sum_{m} a_{n}(t) a_{m}^{*}(t)|n\rangle\langle m|,
$$

que substituído na expressão para a equação de Wigner ficará

$$
\begin{aligned}
& W(x, p, t)=\sum_{n} \sum_{m} a_{n}(t) a_{m}^{*}(t) \frac{1}{\pi \hbar} \int d u\langle x+u \mid n\rangle\langle m \mid x-u\rangle \exp \left(-\frac{2 i p u}{\hbar}\right) \Rightarrow \\
& \Rightarrow W(x, p, t)=\sum_{n} \sum_{m} a_{n}(t) a_{m}^{*}(t) \frac{1}{\pi \hbar} \int d u \phi_{m}^{*}(x-u) \phi_{n}(x+u) \exp \left(-\frac{2 i p u}{\hbar}\right),
\end{aligned}
$$

sendo $\phi_{n}(x)$ auto-funções do hamiltoniano do sistema.

Podemos definir

$$
\omega_{m n}(x, p)=\frac{1}{\pi \hbar} \int d u \phi_{m}^{*}(x-u) \phi_{n}(x+u) \exp \left(-\frac{2 i p u}{\hbar}\right)
$$

e a função de Wigner poderá então ser escrita como

$$
W(x, p, t)=\sum_{n} \sum_{m} a_{n}(t) a_{m}^{*}(t) \omega_{m n}(x, p) .
$$

As funções $\omega_{m n}(x, p)$ formam um sistema ortogonal completo no espaço de fase $(x, p)$, com as seguintes propriedades

$$
\int d x \int d p \omega_{m^{\prime}, n^{\prime}}^{*}(x, p) \omega_{m n}(x, p)=\frac{\delta_{m, m^{\prime}} \delta_{n . n^{\prime}}}{2 \pi \hbar}
$$




$$
\sum_{m} \sum_{n} \omega_{m n}^{*}\left(x^{\prime}, p^{\prime}\right) \omega_{m n}(x, p)=\frac{\delta\left(x^{\prime}-x\right) \delta\left(p^{\prime}-p\right)}{2 \pi \hbar} .
$$

Utilizando estas propriedades podemos encontrar as amplitudes de ocupação de cada função $\omega_{m n}(x, p)$ de uma dada função de Wigner $W(x, p, t)$ :

$$
a_{n}(t) a_{m}^{*}(t)=2 \pi \hbar \int d x \int d p w_{m n}^{*}(x, p) W(x, p, t) .
$$

Através desta fórmula é fácil encontrar a probabilidade de ocupação de um determinado nível de energia de um sistema quântico através de sua função de Wigner. Assim, a probabilidade de que, num determinado instante $t$, o sistema se encontre no nível energético $k$ será

$$
P_{k}(t)=\left|a_{k}(t)\right|^{2}=2 \pi \hbar \int d x \int d p \omega_{k k}^{*}(x, p) W(x, p, t)
$$

\subsection{Modelo Simplificado para Três Curvas de Potencial}

Colisões frias mediadas por fótons ressonantes são processos inerentemente dissipativos porque os pares de átomos colidentes têm tempo suficiente para absorver fótons e emiti-los espontaneamente, perdendo energia irreversivelmente para o reservatório de modos vazios do campo eletromagnético. Por exemplo, consideremos dois átomos de sódio, um no estado fundamental, de simetria $S$, e o outro no primeiro estado excitado, de simetria $P$. Estes átomos interagem assintoticamente de acordo com a interação dipolar ressonante

$$
V(R)= \pm a \hbar \gamma\left(\frac{\lambda_{\nu}}{2 \pi R}\right)^{3}
$$

Aqui, $\lambda_{\nu}$ é o comprimento de onda da transição D2 do sódio, $\gamma$ é a taxa de emissão espontânea do estado excitado em unidades de freqüência angular, $a$ é uma constante adimen- 
sional da ordem da unidade e $R$ é a distância interatômica. Notemos também que esta interação pode ser positiva ou negativa, dependendo da orientação relativa entre os elementos de matriz dos momentos de dipolo elétrico atômicos. Temperaturas realísticas de amostras de átomos aprisionados em armadilhas magneto-ópticas são usualmente da ordem da chamada temperatura de resfriamento Doppler, $T_{D}$, dada por [2]

$$
k_{B} T_{D}=\frac{\hbar \gamma}{2}
$$

onde $k_{B}$ é a constante de Boltzmann. As equações (2.26) e (2.27) mostram que $V(R)$, comparado com a escala natural de energia neste contexto, $k_{B} T_{D}$, é apreciável $(V(R) \sim$ $2 k_{B} T_{D}$ ) quando $R$ é da ordem de $\lambda_{\nu} / 2 \pi$. Esta distância interatômica é cerca de $938 \AA$ para a transição D2 do sódio, mostrando o caráter de longo alcance deste tipo de colisão. Átomos de sódio a $T_{D} \approx 240 \mu \mathrm{K}$ têm uma velocidade de apenas $51 \mathrm{~cm} / \mathrm{s}$, podendo viajar apenas $81 \AA$ durante o tempo de vida de 16 ns do estado excitado. Estes números mostram claramente que a duração de colisões frias, $(938 \AA) /(51 \mathrm{~cm} / \mathrm{s}) \approx 184 \mathrm{~ns}$, é consideravelmente mais longa do que o período médio de cerca de 16 ns dentro do qual a emissão espontânea ocorre. Além do decaimento espontâneo, a repopulação do estado excitado durante a colisão se torna freqüente para intensidades típicas dos feixes laser em armadilhas magneto-ópticas e qualquer descrição teórica completa deste tipo de espalhamento deve ser capaz de tratar esta característica única do regime frio.

Assintoticamente, a energia potencial (2.26) atrativa ou repulsiva corresponde a uma aceleração de um átomo em relação ao outro muito maior do que quando ambos os átomos estão em seus estados fundamentais que, neste caso, são acelerados de acordo com o potencial de Van der Waals $1 / R^{6}$ (atrativo). Consideremos um átomo em seu estado ex- 
citado de simetria $P$ acelerado em direção a outro em seu estado fundamental de simetria $S$. Após ganharem uma certa quantidade de energia cinética relativa, pode haver emissão espontânea pela “quase-molécula." Esta transição radiativa não afeta o movimento relativo dos átomos e, portanto, o efeito deste processo radiativo é produzir dois átomos em seus estados fundamentais com energia cinética relativa maior do que quando ambos se aproximam sem jamais serem acelerados pelo potencial $1 / R^{3}$ da equação (2.26). Se este acréscimo de energia cinética relativa for maior do que a profundidade em energia potencial da armadilha magneto-óptica (tipicamente correspondente à ordem de 1 Kelvin), o par de átomos escapa da nuvem aprisionada e este processo de perda atômica é conhecido como escape radiativo.

A emissão espontânea é um processo aleatório, que pode ocorrer ou não durante um especificado intervalo de tempo. Quando a emissão espontânea deixa de ocorrer para um par de átomos em colisão segundo a interação assintótica (2.26) atrativa durante um período de tempo suficientemente longo, o par atômico pode se aproximar o bastante para que o estado assintótico de dois átomos sofra mudança de estrutura fina. Este fenômeno pode ser descrito da seguinte forma. Suponhamos que o estado da "quase-molécula" no início da colisão corresponda, assintoticamente, a um átomo excitado em seu estado de simetria ${ }^{2} P_{3 / 2}$ e outro átomo em seu estado fundamental de simetria ${ }^{2} S_{1 / 2}$. A curva de potencial atrativa que se correlaciona, assintoticamente, com este estado de dois átomos cruza, inevitavelmente, com a curva de potencial repulsivo que se correlaciona, assintoticamente, ao estado de dois átomos em que um dos átomos está em seu estado excitado de simetria ${ }^{2} P_{1 / 2}$ e o outro, em seu estado fundamental de simetria ${ }^{2} S_{1 / 2}$. Seja este ponto de cruzamento 
$R_{c}$. Na verdade, por causa da interação spin-órbita, o cruzamento em $R_{c}$ é um cruzamento evitado. Assim, há uma probabilidade não nula de que a "quase-molécula" mude de estado eletrônico em torno de $R_{c}$ e passe a sofrer dissociação por causa da interação antiligante deste novo estado interno. Ao se separar, os dois átomos colidentes ganham energia cinética relativa por causa da aceleração sobre o potencial repulsivo (2.26):

$$
{ }^{2} P_{3 / 2}+{ }^{2} S_{1 / 2} \rightarrow{ }^{2} P_{1 / 2}+{ }^{2} S_{1 / 2}+\text { energia. }
$$

Como estamos considerando átomos idênticos, cada átomo recebe o mesmo acréscimo de energia cinética e, para alcalinos, este aumento é suficiente para que o par atômico escape da nuvem confinada. A este fenômeno de perda atômica da armadilha dá-se o nome de colisão de mudança de estrutura fina. Para o sódio, por exemplo, o ganho em energia cinética de cada átomo corresponde a aproximadamente $12 \mathrm{~K}$, mais do que suficiente para escapar de uma armadilha magneto-óptica com profundidade típica da ordem de $1 \mathrm{~K}$ [2].

Estamos interessados em colisões entre átomos alcalinos idênticos aprisionados em uma armadilha magneto-óptica. Como espécie típica, tomemos o sódio. À densidade facilmente obtenível de $10^{10}$ átomos por centímetro cúbico da amostra confinada, estimamos uma distância interatômica média da ordem de $5 \times 10^{4} \AA$. O comprimento de onda térmico é definido por

$$
\lambda_{T}=\frac{h}{\sqrt{2 \pi m k_{B} T}}
$$

onde $m$ é a massa de cada átomo, $h=2 \pi \hbar$ e $T$ é a temperatura da amostra. Este comprimento é da ordem do comprimento de de Broglie para uma partícula de massa $m$ e energia cinética $k_{B} T$. Para o caso do sódio confinado em uma armadilha magneto-óptica típica, tomando $T=T_{D} \approx 240 \mu \mathrm{K}$ encontramos $\lambda_{T} \approx 250 \AA$. Assim, em média, o gás é clássico 
e obedece à estatística de Maxwell-Boltzmann, pois os átomos estão longe do regime de degenerescência quântica em uma armadilha magneto-óptica típica. Desta forma, a grande maioria das colisões são binárias e os átomos colidentes iniciam a colisão apenas quando se aproximam de uma distância da ordem de $\lambda_{\nu} / 2 \pi \approx 1000 \AA$, como discutido acima.

Notemos que estas colisões acontencem em uma região espacial em torno do mínimo do campo magnético quadrupolar introduzido pelas bobinas anti-Helmholtz, como mencionado acima. Para simplificar, todos os trabalhos da literatura que consideram o problema de perdas por colisões frias de átomos confinados [2] desprezam a presença do campo magnético, supondo que a variação deste em torno da origem é desprezível na escala de distâncias em que as colisões ocorrem $(\sim 1000 \AA ̊)$. Considerando que os campos magnéticos em armadilhas magneto-ópticas tipicamente possuem um gradiente da ordem de $10 \mathrm{G} / \mathrm{cm}$, vemos que estes campos variam da ordem de $10^{-4} \mathrm{G}$ em $1000 \AA$ (extensão da região em que uma colisão acontece), implicando uma variação da freqüência de transição atômica por efeito Zeeman menor do que $1 \mathrm{kHz}$, desprezível completamente. Assim, desprezamos o efeito do campo magnético no centro da armadilha magneto-óptica onde a amostra se localiza.

Como há um conjunto de feixes laser presente, há bombeamento óptico dos níveis de energia ressonantes dos átomos distantes entre si e, portanto, quando a colisão é iniciada, as populações dos níveis excitados já são diferentes de zero. O campo elétrico presente se deve aos feixes laser que se encontram no centro da amostra atômica. Todas as polarizações estão presentes e todos os feixes têm a mesma freqüência $\omega_{L}$ (ignorando a largura espectral da ordem de $1 \mathrm{MHz}$ de um feixe laser). Além disso, a intensidade de um feixe laser tipicamente 
utilizado em uma armadilha magneto-óptica é da ordem de $100 \mathrm{~mW} / \mathrm{cm}^{2}$, o que implica em uma densidade de aproximadamente $10^{6}$ fótons por centímetro cúbico. Pelo princípio da correspondência, este campo elétrico é clássico. Por simplicidade consideraremos apenas uma polarização do campo elétrico e escolheremos $E_{q}(\vec{R})$ como a amplitude de apenas um feixe laser se propagando em uma única direção, o que podemos aproximar a uma onda plana. Desprezaremos, ainda, a inclusão de campo quantizado e trataremos o caso em que a repopulação do estado excitado pode ser desprezada, isto é, o caso de baixa intesidade do laser de prova. No presente modelo, consideraremos que apenas dois estados eletrônicos da "quase molécula" são acoplados via interação com o campo de prova.

Como simplificação, consideraremos o caso artificial em que a colisão se dá em uma dimensão, o que não muda o formalismo empregado, que pode ser facilmente generalizado para o caso de três dimensões, o que nos dá o seguinte Hamiltoniano (Apêndice A):

$$
\begin{aligned}
\hat{H}= & \frac{p_{x}^{2}}{2 \mu}+\left[V_{1}(x)\right] \hat{s} \hat{s}^{\dagger}+\left[V_{2}(x)\right] \hat{s}^{\dagger} \hat{s}+\left[V_{3}(x)\right] \hat{\sigma}^{\dagger} \hat{\sigma} \\
& -\hbar \Omega\left\{\left[\exp \left(-i \omega_{L} t\right)\right] \hat{s}^{\dagger}+\left[\exp \left(i \omega_{L} t\right)\right] \hat{s}\right\}+\frac{\hbar \varkappa}{2}\left(\hat{\sigma}^{\dagger}+\hat{\sigma}\right)
\end{aligned}
$$

onde $\hbar \Omega$ é o acoplamento, via interação com o laser, entre os níveis $|1\rangle$ e $|2\rangle$ e $\hbar \varkappa$ o acoplamento entre os níveis de estrutura fina $|2\rangle$ e $|3\rangle$. Mais tarde, no Capítulo 3, será adicionado um acoplamento entre os níveis $|1\rangle$ e $|2\rangle$ na forma de decaimento. 


\subsection{Evolução da Função de Wigner para o Modelo de Três Curvas de Potencial}

Nesta seção buscamos calcular as equações de movimento de Heisenberg relevantes para nosso problema. Para tal seguiremos um procedimento semelhante ao feito por [6]. O cálculo detalhado é feito no Apêndice B.

Acima mostramos que, para uma amostra de sódio aprisionada magneto-opticamente, o comprimento de onda térmico é da ordem de $250 \AA$ e que, portanto, comparado com a distância interatômica média de cerca de $50000 \AA$, implica que o gás obedece à estatística clássica. No entanto, $250 \AA$ não é muito menor do que a distância, da ordem de $1000 \AA$, em que a colisão fria começa. Neste caso, podemos descrever o movimento relativo dos átomos colidentes por uma trajetória clássica? Quão realística uma tal aproximação seria? Um dos principais objetivos desta área de pesquisa é obter respostas a estas questões de maneira quantitativa, inclusive no caso de termos mais do que apenas duas curvas de potencial envolvidas. Neste ponto é que a importância do operador de Wigner se torna aparente, pois esta ferramenta nos permite investigar estas questões de uma maneira muito conveniente. Dado que a dinâmica dos graus de liberdade internos da "quase-molécula" é, por sua própria natureza, puramente quântica e, portanto, não há uma aproximação semiclássica para este aspecto do problema que possamos adotar sem perder informação relevante, devemos ser capazes de introduzir aproximações semiclássicas aos graus de liberdade externos, isto é, ao movimento relativo, da "quase-molécula," sem afetar sua dinâmica quântica interna. O operador de Wigner é a ferramenta adequada para este tratamento, como ilustramos com um operador hamiltoniano simples do tipo 


$$
\hat{H}_{e x}=\frac{\hat{p}_{x}^{2}}{2 \mu}+V_{e x}(\hat{x})
$$

Segue das propriedades do operador de Wigner [6] que este hamiltoniano pode ser expresso como

$$
\hat{H}_{e x}=\int_{-\infty}^{+\infty} d x \int_{-\infty}^{+\infty} d p_{x}\left[\frac{p_{x}^{2}}{2 \mu}+V_{e x}(x)\right] \hat{f}\left(x, p_{x}\right),
$$

implicando equações de movimento de Heisenberg da forma

$$
\left(\frac{\partial}{\partial t}+\frac{p_{x}}{\mu} \frac{\partial}{\partial x}\right) \hat{f}\left(x, p_{x}\right)=\int_{-\infty}^{+\infty} d p_{x}^{\prime} J\left(x, p_{x}-p_{x}^{\prime}\right) \hat{f}\left(x, p_{x}^{\prime}\right),
$$

com

$$
J\left(x, p_{x}\right)=\frac{1}{i \hbar(2 \pi \hbar)} \int_{-\infty}^{+\infty} d \xi\left[V_{e x}\left(x+\frac{\xi}{2}\right)-V_{e x}\left(x-\frac{\xi}{2}\right)\right] \exp \left[-i \frac{p_{x} \xi}{\hbar}\right]
$$

Se expandimos $V_{e x}\left(x \pm \frac{\xi}{2}\right)$ no integrando desta expressão em potências de $\xi, J\left(x, p_{x}\right)$ pode ser expressa em uma série de potências de $\hbar$, que pode ser truncada após termos uma expressão até a ordem que desejarmos. Assim, por exemplo, até ordem zero de $\hbar$ obtemos

$$
J\left(x, p_{x}\right)=\frac{\partial V_{e x}(x)}{\partial x} \frac{\partial \delta\left(p_{x}\right)}{\partial p_{x}},
$$

resultando na equação de Liouville clássica para o operador de Wigner:

$$
\left(\frac{\partial}{\partial t}+\frac{p_{x}}{\mu} \frac{\partial}{\partial x}\right) \hat{f}\left(x, p_{x}\right)=\frac{\partial V_{e x}(x)}{\partial x} \frac{\partial \hat{f}\left(x, p_{x}\right)}{\partial p_{x}} .
$$

Então, se utilizamos a expansão dos operadores $\hat{p}_{x}^{2} / 2 \mu$ e $V_{1,2,3}(\hat{x})$ em termos do operador de Wigner na equação (2.30), podemos examinar aproximações semiclássicas do movimento relativo até qualquer ordem de $\hbar$, mantendo a dinâmica quântica interna intacta. É também importante dizer que, nesta aproximação os $\hbar$ 's do estado inicial, se presentes, não são afetados pela evolução aproximada. Por esta razão adotamos a abordagem em 
termos do operador de Wigner. Vemos aqui que, na ausência da dinâmica quântica dos graus de liberdade internos da Equação (2.30), por exemplo, o limite $\hbar \rightarrow 0$ na expansão de $J$ resulta na dinâmica clássica para os graus de liberdade translacionais. Tivéssemos continuado a expansão para ordens maiores de $\hbar$, teríamos obtido correções quânticas ao movimento translacional.

A evolução do operador de Wigner para (2.30), como calculado no Apêndice B, resulta no seguinte conjunto de equações:

$$
\begin{aligned}
&\left(\frac{\partial}{\partial t}+\frac{p}{\mu} \frac{\partial}{\partial x}\right) \rho_{11}(x, p, t)= V_{g}^{\prime}(x) \frac{\partial}{\partial p} \rho_{11}(x, p, t)+ \\
&+i \Omega\left[-\rho_{12}(x, p, t)+\rho_{21}(x, p, t)\right] \\
&\left(\frac{\partial}{\partial t}+\frac{p}{\mu} \frac{\partial}{\partial x}\right) \rho_{22}(x, p, t)= V_{e}^{\prime}(x) \frac{\partial}{\partial p} \rho_{22}(x, p, t)+ \\
&+i \Omega\left[\rho_{12}(x, p, t)-\rho_{21}(x, p, t)\right]+ \\
&+\frac{i \varkappa}{2}\left[\rho_{23}(x, p, t)-\rho_{32}(x, p, t)\right] \\
&\left(\frac{\partial}{\partial t}+\frac{p}{\mu} \frac{\partial}{\partial x}\right) \rho_{33}(x, p, t)= V_{i}^{\prime}(x) \frac{\partial}{\partial p} \rho_{33}(x, p, t)+ \\
&+\frac{i \varkappa}{2}\left[\rho_{32}(x, p, t)-\rho_{23}(x, p, t)\right] \\
&\left(\frac{\partial}{\partial t}+\frac{p}{\mu} \frac{\partial}{\partial x}\right) \rho_{21}(x, p, t)=\frac{1}{2}\left[V_{e}^{\prime}(x)+V_{g}^{\prime}(x)\right] \frac{\partial}{\partial p} \rho_{21}(x, p, t)+ \\
&+i\left[\Delta-\frac{V_{e}(x)-V_{g}(x)}{\hbar}\right] \rho_{21}(x, p, t)+
\end{aligned}
$$




$$
\begin{aligned}
& +i \Omega\left[\rho_{11}(x, p, t)-\rho_{22}(x, p, t)\right]- \\
& -\frac{i \varkappa}{2} \rho_{31}(x, p, t)
\end{aligned}
$$

$$
\begin{aligned}
\left(\frac{\partial}{\partial t}+\frac{p}{\mu} \frac{\partial}{\partial x}\right) \rho_{12}(x, p, t)= & \frac{1}{2}\left[V_{e}^{\prime}(x)+V_{g}^{\prime}(x)\right] \frac{\partial}{\partial p} \rho_{12}(x, p, t)+ \\
& -i\left[\Delta-\frac{V_{e}(x)-V_{g}(x)}{\hbar}\right] \rho_{12}(x, p, t)+ \\
& +i \Omega\left[\rho_{22}(x, p, t)-\rho_{11}(x, p, t)\right]+ \\
& +\frac{i \varkappa}{2} \rho_{13}(x, p, t)
\end{aligned}
$$

$$
\begin{aligned}
\left(\frac{\partial}{\partial t}+\frac{p}{\mu} \frac{\partial}{\partial x}\right) \rho_{23}(x, p, t)= & \frac{1}{2}\left[V_{e}^{\prime}(x)+V_{i}^{\prime}(x)\right] \frac{\partial}{\partial p} \rho_{23}(x, p, t)+ \\
& -i\left[\varkappa-\frac{V_{i}(x)-V_{e}(x)}{\hbar}\right] \rho_{23}(x, p, t)+ \\
& +\frac{i \varkappa}{2}\left[\rho_{33}(x, p, t)-\rho_{22}(x, p, t)\right]+ \\
& +i \Omega \rho_{13}(x, p, t)
\end{aligned}
$$

$$
\begin{aligned}
\left(\frac{\partial}{\partial t}+\frac{p}{\mu} \frac{\partial}{\partial x}\right) \rho_{32}(x, p, t)= & \frac{1}{2}\left[V_{e}^{\prime}(x)+V_{i}^{\prime}(x)\right] \frac{\partial}{\partial p} \rho_{32}(x, p, t)+ \\
& +i\left[\varkappa+\frac{V_{e}(x)-V_{i}(x)}{\hbar}\right] \rho_{32}(x, p, t)+ \\
& +\frac{i \varkappa}{2}\left[\rho_{22}(x, p, t)-\rho_{33}(x, p, t)\right]- \\
& -i \Omega \rho_{31}(x, p, t)
\end{aligned}
$$

$$
\left(\frac{\partial}{\partial t}+\frac{p}{\mu} \frac{\partial}{\partial x}\right) \rho_{31}(x, p, t)=\frac{1}{2}\left[V_{g}^{\prime}(x)+V_{i}^{\prime}(x)\right] \frac{\partial}{\partial p} \rho_{31}(x, p, t)+
$$




$$
\begin{aligned}
& +i\left[\Delta+\varkappa+\frac{V_{g}(x)-V_{i}(x)}{\hbar}\right] \rho_{31}(x, p, t)+ \\
& -\frac{i \varkappa}{2} \rho_{21}(x, p, t)+i \Omega \rho_{32}(x, p, t),
\end{aligned}
$$

$$
\begin{aligned}
\left(\frac{\partial}{\partial t}+\frac{p}{\mu} \frac{\partial}{\partial x}\right) \rho_{13}(x, p, t)= & \frac{1}{2}\left[V_{g}^{\prime}(x)+V_{i}^{\prime}(x)\right] \frac{\partial}{\partial p} \rho_{13}(x, p, t)+ \\
& -i\left[\Delta+\varkappa+\frac{V_{g}(x)-V_{i}(x)}{\hbar}\right] \rho_{13}(x, p, t)+ \\
& -\frac{i \varkappa}{2} \rho_{12}(x, p, t)+i \Omega \rho_{23}(x, p, t) .
\end{aligned}
$$

onde os $\rho_{i j}$ são as funções de Wigner conforme definido no Apêndice B e $V_{g}(x)=V_{1}(x)$, $V_{e}(x)=V_{2}(x)-\hbar \omega_{0}$ e $V_{i}(x)=V_{3}(x)-\hbar \omega_{0}+\hbar \varkappa$ e $\Delta=\omega_{L}-\omega_{0}$

Note que o presente modelo não inclui termos de decaimento, os quais podem ser incluídos futuramente com a inclusão de termos dependentes de um fator $\Gamma$.

Podemos reescrever as equações acima na forma geral:

$$
\frac{\partial \rho_{i j}(x, p, t)}{\partial t}=-\frac{p}{\mu} \frac{\partial \rho_{i j}(x, p, t)}{\partial x}+F_{i j}(x) \frac{\partial \rho_{i j}(x, p, t)}{\partial p}+\frac{1}{i \hbar} \sum_{k}\left(H_{i k} \rho_{k j}-H_{k j} \rho_{i k}\right)
$$

$\operatorname{com} i=1, \ldots, 3$ e $j=1, \ldots, 3$, onde $F_{11}(x)=V_{g}^{\prime}(x), F_{22}(x)=V_{e}^{\prime}(x), F_{33}(x)=V_{i}^{\prime}(x)$, $F_{12}(x)=F_{21}(x)=\frac{1}{2}\left[V_{e}^{\prime}(x)+V_{g}^{\prime}(x)\right], F_{13}(x)=F_{31}(x)=\frac{1}{2}\left[V_{g}^{\prime}(x)+V_{i}^{\prime}(x)\right] \mathrm{e}$ $F_{23}(x)=F_{32}(x)=\frac{1}{2}\left[V_{e}^{\prime}(x)+V_{i}^{\prime}(x)\right]$. A resolução deste sistema de equações diferenciais deverá ser feita através do método do operador partido. 


\section{Capítulo 3: Desenvolvimento do Algorítmo}

\subsection{Forma Matricial}

Utilizando a representação de Heisenberg para fazermos a evolução temporal do operador de Wigner [3], segundo o Hamiltoniano deste problema, seguindo [6], e ainda, tomando apenas os termos até ordem zero em $\hbar$, na dinâmica dos graus de liberdade translacionais, chegamos ao seguinte sistema de equações diferenciais acopladas, calculadas no Apêndice B, que podem ser escritas matricialmente como:

$$
\frac{\partial \hat{U}}{\partial t}=\hat{L} \hat{U}+\hat{M} \hat{U}
$$

onde o operador $\hat{L}$ faz a evolução externa do sistema que foi tomada como clássica na aproximação feita no Apêndice B, e é denotado por:

$$
\hat{L}=-(\hat{V}-\hat{F}),
$$

onde $\hat{V}$ e $\hat{F}$ são dados por:

$$
\hat{V}=\frac{p}{\mu} \hat{I}_{9} \frac{\partial}{\partial x},
$$

onde $\hat{I}_{9}$ é a matriz identidade $9 \times 9 \mathrm{e}$ 


$$
\hat{F}=\left(\begin{array}{ccccccccc}
F_{11} & 0 & 0 & 0 & 0 & 0 & 0 & 0 & 0 \\
0 & F_{12} & 0 & 0 & 0 & 0 & 0 & 0 & 0 \\
0 & 0 & F_{13} & 0 & 0 & 0 & 0 & 0 & 0 \\
0 & 0 & 0 & F_{21} & 0 & 0 & 0 & 0 & 0 \\
0 & 0 & 0 & 0 & F_{22} & 0 & 0 & 0 & 0 \\
0 & 0 & 0 & 0 & 0 & F_{23} & 0 & 0 & 0 \\
0 & 0 & 0 & 0 & 0 & 0 & F_{31} & 0 & 0 \\
0 & 0 & 0 & 0 & 0 & 0 & 0 & F_{32} & 0 \\
0 & 0 & 0 & 0 & 0 & 0 & 0 & 0 & F_{33}
\end{array}\right) \frac{\partial}{\partial p}
$$

onde, como já definido anteriormente, $F_{11}(x)=V_{g}^{\prime}(x), F_{22}(x)=V_{e}^{\prime}(x), F_{33}(x)=$ $V_{i}^{\prime}(x), F_{12}(x)=F_{21}(x)=\frac{1}{2}\left[V_{e}^{\prime}(x)+V_{g}^{\prime}(x)\right], F_{13}(x)=F_{31}(x)=\frac{1}{2}\left[V_{g}^{\prime}(x)+V_{i}^{\prime}(x)\right]$ e $F_{23}(x)=F_{32}(x)=\frac{1}{2}\left[V_{e}^{\prime}(x)+V_{i}^{\prime}(x)\right]$.

$\hat{U}$ é a matriz das funções de Wigner dos três estados e suas respectivas coerências.

$$
\hat{U}=\left(\begin{array}{c}
\rho_{11}(x, p, t) \\
\rho_{12}(x, p, t) \\
\rho_{13}(x, p, t) \\
\rho_{21}(x, p, t) \\
\rho_{22}(x, p, t) \\
\rho_{23}(x, p, t) \\
\rho_{31}(x, p, t) \\
\rho_{32}(x, p, t) \\
\rho_{33}(x, p, t)
\end{array}\right)
$$

Por fim, $\hat{M}$ é o que faz a evolução interna do sistema, que continua sendo puramente quântica, contendo os acoplamentos entre os níveis e gerando as possíveis transições de níveis: 


$$
\hat{M}=\left(\begin{array}{ccccccccc}
0 & -i \Omega & 0 & i \Omega & 0 & 0 & 0 & 0 & 0 \\
-i \Omega & -i \Delta_{12} & i \frac{\chi}{2} & 0 & i \Omega & 0 & 0 & 0 & 0 \\
0 & i \frac{\chi}{2} & i \Delta_{13} & 0 & 0 & i \Omega & 0 & 0 & 0 \\
i \Omega & 0 & 0 & i \Delta_{12} & -i \Omega & 0 & -i \frac{\chi}{2} & 0 & 0 \\
0 & i \Omega & 0 & -i \Omega & 0 & i \frac{\chi}{2} & 0 & -i \frac{\chi}{2} & 0 \\
0 & 0 & i \Omega & 0 & i \frac{\chi}{2} & i \Delta_{23} & 0 & 0 & -i \frac{\chi}{2} \\
0 & 0 & 0 & -i \frac{\chi}{2} & 0 & 0 & -i \Delta_{13} & -i \Omega & 0 \\
0 & 0 & 0 & 0 & -i \frac{\chi}{2} & 0 & -i \Omega & -i \Delta_{23} & i \frac{\chi}{2} \\
0 & 0 & 0 & 0 & 0 & -i \frac{\chi}{2} & 0 & i \frac{\chi}{2} & 0
\end{array}\right)
$$

onde $\Delta_{12}, \Delta_{13}$ e $\Delta_{23}$ são as diferenças energéticas entre os níveis e são definidos abaixo:

$$
\begin{aligned}
& \Delta_{12}=\left[\Delta-\frac{\left(V_{e}-V_{g}\right)}{\hbar}\right], \\
& \Delta_{13}=\left[\Delta+\chi-\frac{\left(V_{g}-V_{i}\right)}{\hbar}\right], \\
& \Delta_{23}=\left[\chi-\frac{\left(V_{i}-V_{e}\right)}{\hbar}\right] .
\end{aligned}
$$

\subsection{Resolução das Equações Diferenciais}

\subsubsection{Operador Partido com Trajetórias}

O método escolhido para resolver o sistema de equações diferenciais (3.41), mostrado acima, foi o método do operador partido. Segundo tal método o sistema de equações diferenciais,

$$
\frac{\partial \hat{U}}{\partial t}=\hat{L} \hat{U}+\hat{M} \hat{U}
$$

pode ser decomposto em 


$$
\begin{aligned}
& \frac{\partial \hat{U}}{\partial t}=\hat{L} \hat{U} \\
& \frac{\partial \hat{U}}{\partial t}=\hat{M} \hat{U}
\end{aligned}
$$

desde que saibamos como cada operador, $\hat{L}$ e $\hat{M}$, evolui segundo as equações (3.47) e (3.48). Assim, o método consiste em dizer que se $E_{\hat{L}}(\hat{U}(0), \Delta t)$ é uma maneira de evoluirmos (3.47) de um tempo $t=0$ até um tempo $t=\Delta t$ e $E_{\hat{M}}(\hat{U}(0), \Delta t)$ é uma maneira de evoluirmos (3.48), também de $t=0$ até $t=\Delta t$, então, a evolução de (3.41), dado seu valor em $t=0$ é obtida da seguinte maneira:

$$
\hat{U}(\Delta t)=E_{\hat{M}}\left[E_{\hat{L}}(\hat{U}(0), \Delta t), \Delta t\right]
$$

Ou seja, num primeiro passo desprezamos o operador $\hat{M}$ e evoluímos apenas com $\hat{L}$ de $t=0$ até $t=\Delta t$. O resultado obtido desta forma será utilizado como condição inicial para a próxima evolução que se dará em $\hat{M}$, desprezando-se, então, o $\hat{L}$. Como resultado, temos a solução de (3.41) a menos de um erro da ordem de $\left(\Delta t^{2}\right)$ a cada passo. Este processo é repetido tantas vezes quanto se queira, sempre utilizando como valor inicial o resultado do passo anterior.

Este é um método particularmente útil, quando se conhecem soluções analíticas para (3.47) e (3.48). Em nosso caso, no entanto, por não possuirmos soluções analíticas de tais equações utilizaremos métodos numéricos na resolução das mesmas. 


\subsubsection{Evolução com $\hat{L}$}

A equação (3.47), em nosso caso, é escrita como um sistema de nove equações diferenciais, visto que $\hat{L}$ e $\hat{U}$ são dados por (3.42) e (3.45). Essas nove equações são da forma:

$$
\frac{\partial \rho(x, p, t)}{\partial t}+\frac{p}{m} \frac{\partial \rho(x, p, t)}{\partial x}-V^{\prime}(x) \frac{\partial \rho(x, p, t)}{\partial p}=0
$$

que nada mais é do que a equação de Liouville.

Sendo $(x, p, t)$ no espaço de fase, usando a notação

$$
\begin{aligned}
\rho_{1}\left(x_{1}, x_{2}, x_{3}\right) & \equiv \frac{\partial}{\partial x_{1}} \rho\left(x_{1}, x_{2}, x_{3}\right) \\
\rho_{2}\left(x_{1}, x_{2}, x_{3}\right) & \equiv \frac{\partial}{\partial x_{2}} \rho\left(x_{1}, x_{2}, x_{3}\right) \\
\rho_{3}\left(x_{1}, x_{2}, x_{3}\right) & \equiv \frac{\partial}{\partial x_{3}} \rho\left(x_{1}, x_{2}, x_{3}\right)
\end{aligned}
$$

para qualquer $\left(x_{1}, x_{2}, x_{3}\right)$. Para encontrar $\rho(x, p, t)$ tal que:

$$
\rho_{3}(x, p, t)+\frac{p}{\mu} \rho_{1}(x, p, t)-V \prime(x) \rho_{2}(x, p, t)=0
$$

para qualquer $(x, p, t)$. Consideramos, então, $x_{c l}(\lambda), p_{c l}(\lambda)$ e $t_{c l}(\lambda)$ tais que:

$$
\begin{aligned}
\frac{d}{d \lambda} x_{c l}(\lambda) & =\frac{1}{\mu} p_{c l}(\lambda) \\
\frac{d}{d \lambda} p_{c l}(\lambda) & =-V^{\prime}\left(x_{c l}(\lambda)\right) \\
\frac{d}{d \lambda} t_{c l}(\lambda) & =1
\end{aligned}
$$


Assim,

$$
\begin{aligned}
\frac{d}{d \lambda} \rho\left(x_{c l}(\lambda), p_{c l}(\lambda), t_{c l}(\lambda)\right)= & {\left[\frac{\partial}{\partial t_{c l}(\lambda)}+\frac{d x_{c l}(\lambda)}{d \lambda} \frac{\partial}{\partial x_{c l}(\lambda)}+\frac{d p_{c l}(\lambda)}{d \lambda} \frac{\partial}{\partial p_{c l}(\lambda)}\right] \times } \\
& \times \rho\left(x_{c l}(\lambda), p_{c l}(\lambda), t_{c l}(\lambda)\right),
\end{aligned}
$$

ou seja:

$$
\begin{aligned}
\frac{d}{d \lambda} \rho\left(x_{c l}(\lambda), p_{c l}(\lambda), t_{c l}(\lambda)\right)= & \rho_{3}\left(x_{c l}(\lambda), p_{c l}(\lambda), t_{c l}(\lambda)\right)+ \\
& +\frac{p_{c l}(\lambda)}{\mu} \rho_{1}\left(x_{c l}(\lambda), p_{c l}(\lambda), t_{c l}(\lambda)\right)+ \\
& -V^{\prime}\left(x_{c l}(\lambda)\right) \rho_{2}\left(x_{c l}(\lambda), p_{c l}(\lambda), t_{c l}(\lambda)\right)
\end{aligned}
$$

Como a Eq. (3.50) vale para quaisquer $(x, p, t)$, então, vale para $\left(x_{c l}(\lambda), p_{c l}(\lambda), t_{c l}(\lambda)\right)$ e portanto,

$$
\frac{d}{d \lambda} \rho\left(x_{c l}(\lambda), p_{c l}(\lambda), t_{c l}(\lambda)\right)=0
$$

o que nos leva a

$$
\rho\left(x_{c l}(\lambda), p_{c l}(\lambda), t_{c l}(\lambda)\right)=\rho\left(x_{c l}(0), p_{c l}(0), t_{c l}(0)\right)
$$

escolhendo $\left(x_{c l}(0), p_{c l}(0), t_{c l}(0)\right) \equiv(x, p, t)$, temos

$$
\begin{gathered}
\frac{d}{d \lambda} t_{c l}(\lambda)=1 \Longrightarrow t_{c l}(\lambda)=t+\lambda \\
\therefore \rho\left(x_{c l}(\lambda), p_{c l}(\lambda), t+\lambda\right)=\rho\left(x_{c l}(0), p_{c l}(0), t_{c l}(0)\right),
\end{gathered}
$$

para qualquer $\lambda$. Assim, escolhendo $\lambda=-t$, vem:

$$
\rho(x, p, t)=\rho\left(x_{c l}(-t), p_{c l}(-t), 0\right)
$$




$$
\begin{aligned}
& \frac{d}{d(-t)} x_{c l}(-t)=\frac{1}{\mu} p_{c l}(-t) \\
& \frac{d}{d(-t)} p_{c l}(-t)=-V^{\prime}\left(x_{c l}(-t)\right) .
\end{aligned}
$$

assim, mostramos que basta resolvermos as equações (3.51) e (3.52), para obtermos o reultado de (3.49). Tais equações são equações diferenciais acopladas e descrevem uma trajetória clássica. Por esta razão, o método foi rebatizado como método do operador partido com trajetórias.

Uma vez que essas equações descrevem uma trajetória clássica e que para qualquer tempo $t, \rho(x, p, t)$ é constante numa trajetória clássica, para determinarmos $\rho(x, p, t)$ basta determinarmos $\rho(x(-t), p(-t), 0)$.

Para tal, basta regredirmos $x(0) \Rightarrow x(-t)$ e $p(0) \Rightarrow p(-t)$. Esta regressão será feita pelo método de Runge-Kutta de quarta ordem [10], por ser este um método bastante simples e adequado na resolução de sistemas acoplados.

Uma vez conhecidos os valores $x(-t)$ e $p(-t)$ é ainda necessário se fazer uma interpolação para obter $\rho(x(-t), p(-t), 0)$. Optamos por fazer uma interpolação bidimensional simples, pois a função $\rho(x, p, t)$ deve ser suave.

\subsubsection{Evolução com $\hat{M}$}

A evolução com $\hat{M}$ será feita utilizando novamente o método do operador partido. Assim, separaremos o operador $\hat{M}$ em outros dois operadores, que possuem solução analítica. Um deles, contendo os termos de acoplamento entre os níveis $V_{e}$ e $V_{g}$, que são acoplados via laser de prova, e o outro contendo os acoplamentos entre os níveis de estrutura fina $V_{e}$ e $V_{i}$. 
A evolução em $\hat{M}$ é dada pela equação

$$
\frac{\partial \hat{U}}{\partial t}=\hat{M} \hat{U}
$$

sendo $\hat{M}$ definido como em (3.46). Podemos então separar o operador $\hat{M}=\hat{M}_{12}+\hat{M}_{23}$. Assim, a equação (3.48) se torna:

$$
\frac{\partial \hat{U}}{\partial t}=\left(\hat{M}_{12}+\hat{M}_{23}\right) \hat{U}
$$

Utilizando o operador partido:

$$
\begin{aligned}
& \frac{\partial \hat{U}}{\partial t}=\hat{M}_{12} \hat{U} \\
& \frac{\partial \hat{U}}{\partial t}=\hat{M}_{23} \hat{U} .
\end{aligned}
$$

Estas equações possuem solução analítica simples. Tais soluções estão apresentadas no Apêndice C.

É neste ponto que podemos acrescentar termos de decaimento no problema, onde, ao invés de separarmos o sistema em apenas duas partes, acrescentamos outro operador que provoca decaimento que será chamado $\hat{M}_{21}^{\Gamma}$, uma vez que o decaimento ocorre apenas do estado excitado para o estado fundamental. Assim,

$$
\frac{\partial \hat{U}}{\partial t}=\left(\hat{M}_{12}+\hat{M}_{23}+\hat{M}_{21}^{\Gamma}\right) \hat{U}
$$

Dessa maneria, teremos também a equação para o decaimento:

$$
\frac{\partial \hat{U}}{\partial t}=\hat{M}_{21}^{\Gamma} \hat{U}
$$

cuja solução analítica, que é trivial, também é apresentada no Apêndice C. 


\subsection{Considerações sobre o Algoritmo}

Como pudemos ver, o método do operador partido com trajetórias nos possibilita separar a equação que descreve a evolução temporal da função de Wigner em duas partes: uma parte clássica, descrita pela equação de Liouville, e outra quântica, que descreve a dinâmica interna do problema. Através deste método é possível obter o valor de cada componente da função de Wigner em todo espaço de fase e, para cada instante de tempo, desde que evoluamos alternadamente a parte clássica e cada uma das três partes da equação quântica, para cada intervalo de tempo, até obtermos a evolução de um tempo $t_{i}$ até um tempo $t_{f}$.

Desta maneira podemos acompanhar a evolução do sistema durante todo o processo, inclusive na região de cruzamento onde esperamos que ocorra a mudança de estrutura fina.

É importante salientar que foi necessário fazer uma mudança de coordenadas nos parâmetros envolvidos no problema, uma vez que seria impossível trabalhar computacionalmente com grandezas de ordem muito pequena, como, por exmplo, no caso de $\hbar$, que no CGS teria ordem de $10^{-27}$.

Segue uma descrição, passo a passo, do programa desenvolvido por nós em FORTRAN 90 para o cálculo de tais equações, utilizando os métodos descritos acima.

- Em primeiro lugar determinamos os valores iniciais $x_{i}, p_{i}$ e $t_{i}$ e finais $x_{f}, p_{f}$ e $t_{f}$, para sabermos o intervalo no qual estaremos trabalhando;

- Determinamos, a seguir, os passos $\Delta x, \Delta p$ e $\Delta t$, com os quais evoluiremos nossas coordenadas espaciais e temporais, obedecendo os valores de precisão desejados 
sem, no entanto, ignorar as limitações computacionais de maneira a não tornar o cálculo demasiadamente dispendioso;

- Em seguida determinamos uma malha no espaço de fase. É necessário ter o cuidado de escolher um número de pontos satisfatório para descrever uma curva suave, sempre levando em conta a capacidade de memória do computador;

- Calculamos os valores numéricos de $\Delta, \Omega, \varkappa, \hbar$, bem como os potenciais e demais parâmetros envolvidos para o caso de representarmos átomos de sódio;

- Enfim, determinamos o valor inicial das funções $\rho_{\mu \nu}(x(0), p(0), 0)$ como uma distribuição gaussiana, como será mostrado no próximo capítulo;

- Fizemos, então, a evolução em $\hat{L}$ de $\rho_{\mu \nu}(x(0), p(0), 0) \Rightarrow \rho_{\mu \nu}(x(\Delta t), p(\Delta t), \Delta t)$, que foi tomada como condição inicial da etapa seguinte;

- Em seguida fizemos a evolução em $\hat{M}_{12}$ de $\rho_{\mu \nu}(x(\Delta t), p(\Delta t), \Delta t) \Rightarrow$ $\bar{\rho}_{\mu \nu}^{12}(x(\Delta t), p(\Delta t), \Delta t)$, que foi tomada como condição inicial da etapa seguinte;

- Em seguida fizemos a evolução em $\hat{M}_{23}$ de $\bar{\rho}_{\mu \nu}^{12}(x(\Delta t), p(\Delta t), \Delta t) \Rightarrow$ $\bar{\rho}_{\mu \nu}^{23}(x(\Delta t), p(\Delta t), \Delta t)$, que foi tomada como condição inicial da etapa seguinte;

- Em seguida fizemos a evolução em $\hat{M}_{21}^{\Gamma}$ de $\bar{\rho}_{\mu \nu}^{23}(x(\Delta t), p(\Delta t), \Delta t) \Rightarrow$ $\bar{\rho}_{\mu \nu}^{\Gamma}(x(\Delta t), p(\Delta t), \Delta t)$, que foi tomada como condição inicial da etapa seguinte;

- Assim, os quatro útimos passos são repetidos até que se obtenha $\rho_{\mu \nu}\left(x, p, t_{f}\right)$; 
- A saída de dados é feita de maneira a obtermos também valores intermediários de $\rho_{\mu \nu}$; por exemplo, a cada $n \Delta t$ transcorridos. Assim, podemos acompanhar a evolução das funções $\rho_{\mu \nu}$ e, a partir daí, estimar a taxa em que a mudança de estrutura fina e o decaimento ocorrem. 


\section{Capítulo 4: Resultados e Discussões}

Neste capítulo apresentaremos os principais resultados obtidos com a resolução numérica das equações diferenciais mostradas no Capítulo 2. Os resultados aqui apresentados estarão com as unidades transformadas para um novo sistema de unidades, uma vez que não estaríamos aptos a trabalhar computacionalmente com grandezas de ordens muito baixas, sendo assim necessário adaptar nosso sistema de unidades. Os fatores de conversão desse novo sistema se encontram a seguir. A mudança de unidades foi feita de maneira a termos

$$
\left\{\begin{array}{c}
\hbar=1 \\
\lambda_{T}=2
\end{array}\right.
$$

Levando em conta que dois átomos de sódio estão colidindo, os fatores de conversão para o novo sistema de unidades são:

\begin{tabular}{|ccc|}
\hline & Unidades Transformadas & CGS \\
Posição & 1 (unid.) & $\sim 1,7 \times 10^{-6} \mathrm{~cm}$ \\
Massa & $1($ unid. $)$ & $\sim 3,8 \times 10^{-25} \mathrm{~g}$ \\
Momentum & $1($ unid. $)$ & $\sim 6,3 \times 10^{-22} \mathrm{~cm} / \mathrm{s}$ \\
Tempo & $1($ ns $)$ & $10^{-9} \mathrm{~s}$ \\
Energia & $1($ unid. $)$ & $\sim 1,1 \times 10^{-18} \mathrm{erg}$ \\
\hline
\end{tabular}

Como dito anteriormente, nosso modelo foi feito fazendo uso de três curvas de potencial que são as seguintes:

$$
\begin{gathered}
V_{g}(x)=0 \\
V_{e}(x)=4 \varepsilon\left[\left(\frac{\sigma}{x}\right)^{6}-\left(\frac{\sigma}{x}\right)^{3}\right]-\hbar \Delta
\end{gathered}
$$

e

$$
V_{i}(x)=\frac{C_{3}}{x^{3}}-\hbar \Delta-\Delta E_{f s} .
$$




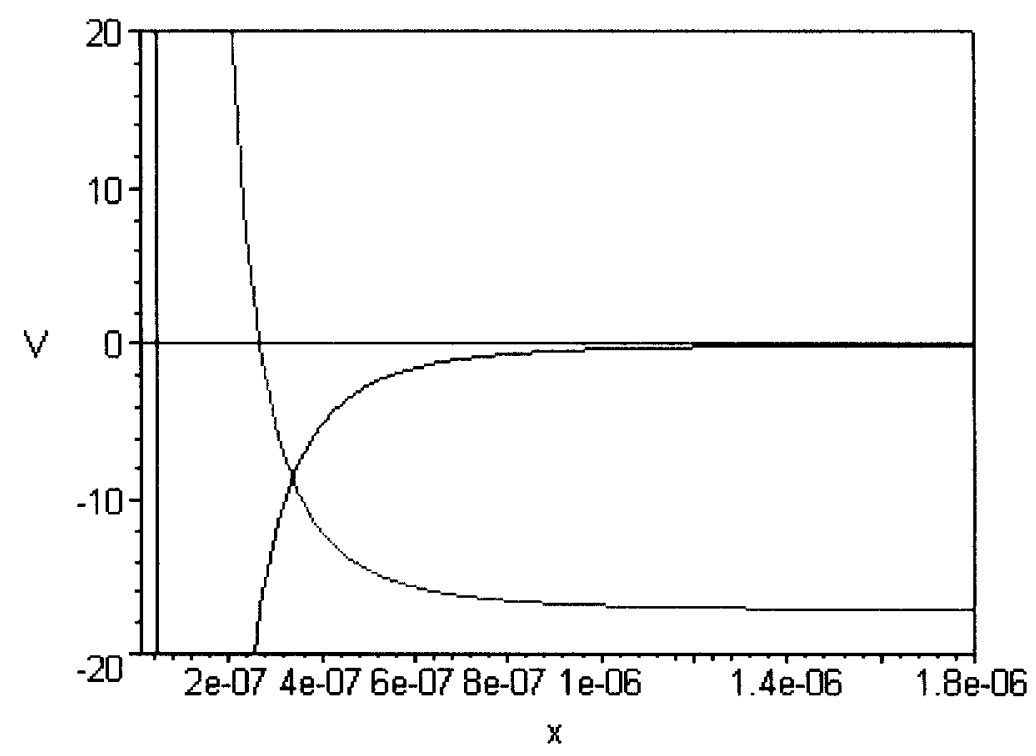

1.Curvas de potencial.

com os seguintes valores no CGS:

$$
\left\{\begin{array}{c}
\varepsilon \approx 1,49 \times 10^{-13} \mathrm{erg}, \\
\sigma \approx 4,76 \times 10^{-8} \mathrm{~cm}, \\
\Delta \approx-3,14 \times 10^{9} \mathrm{~s}^{-1} \\
C_{3} \approx 6,46 \times 10^{-35} \mathrm{erg} . \mathrm{cm}^{3} \\
\Delta E_{f s} \approx 3,41 \times 10^{-15} \mathrm{erg} .
\end{array}\right.
$$

Especificamente no caso do sódio as curvas $V_{e}$ e $V_{g}$ se cruzam aproximadamente nos $508 a_{0}$ e as curvas $V_{i}$ e $V_{e}$ se cruzam nos $63 a_{0}$. Para valores menores que $50 a_{0}$ as curvas acima deixam de ter um comportamento suave, uma vez que ambas $V_{i}$ e $V_{e}$ são singulares na origem. Para evitar uma variação brusca de energia, à medida que se evolui um intervalo $\Delta x$ pré-determinado, quando $x \rightarrow 0$, inserimos no algoritmo uma barreira de potencial infinita próxima aos $60 a_{0}$, o que causa uma reflexão total da onda, sem perdas [11]. Assim 
sendo, a inclusão da barreira de potencial não prejudica a observação dos resultados uma vez que a função de Wigner passa pelos dois cruzamentos relevantes antes de ser refletida.

Como condição inicial para a função de Wigner usamos uma distribuição gaussiana da forma

$$
\rho(x, p, 0)=\frac{1}{\pi \hbar} \exp \left[-\frac{\left(x-x_{0}\right)^{2}}{\sigma^{2}}\right] \exp \left[-\frac{\sigma^{2}\left(p-p_{0}\right)^{2}}{\hbar^{2}}\right]
$$

que, como podemos ver, obedece ao princípio da incerteza de Heisenberg, já que

$$
\Delta x \Delta p \geqslant \hbar
$$

Esta distribuição inicial é centrada em $x_{0}=8($ unid.) o que corresponde a aproximadamente $2512 a_{0}$, e $p_{0} \cong-1.77$ (unid.) o que corresponderia a aproximadamente $-1.12 \times$ $10^{-21} \mathrm{~cm} / \mathrm{s}$, que é negativo pois a distribuição anda em direção à origem de $x$. 


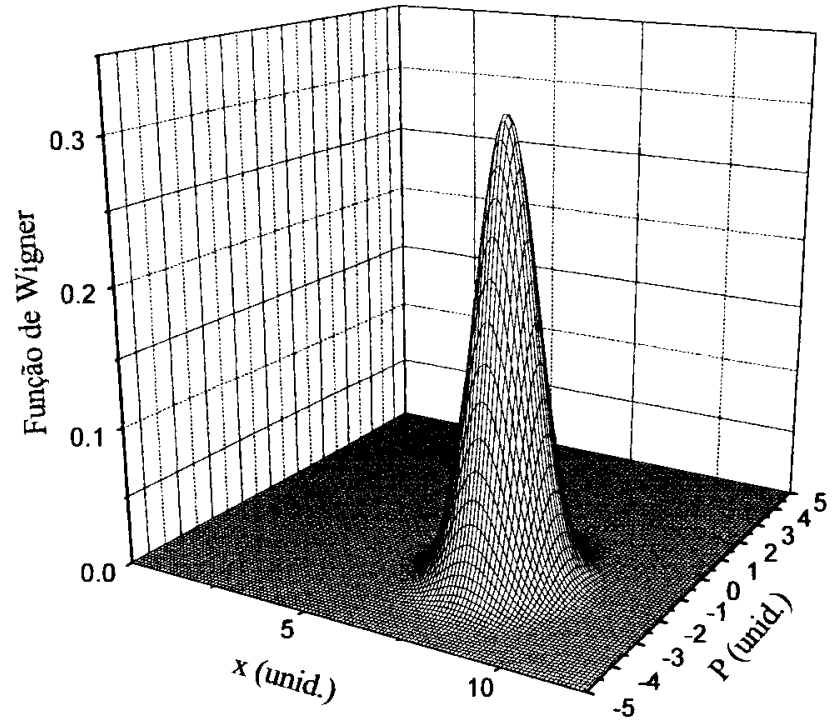

2.Gráfico da distribuição inicial.

O gráfico acima mostra a distribuição inicial $\rho(x, p, 0)$ com os parâmetros fixados acima. 


\subsection{Sistema sem decaimento}

Por enquanto não levaremos em conta o decaimento. Assim, começaremos nosso sistema apenas com o estado fundamental $V_{g}$ populado:

$$
\begin{aligned}
& \rho_{11}(x, p, 0)=\rho(x, p, 0) \\
& \rho_{22}(x, p, 0)=0 \\
& \rho_{33}(x, p, 0)=0 .
\end{aligned}
$$

Faremos aqui a análise da dinâmica das populações dos três estados. As populações são dadas por:

$$
\begin{aligned}
& P_{1}(t)=\int d p \int d x \rho_{11}(x, p, t), \\
& P_{2}(t)=\int d p \int d x \rho_{22}(x, p, t), \\
& P_{3}(t)=\int d p \int d x \rho_{33}(x, p, t) .
\end{aligned}
$$

Aqui utilizamos

$$
\left\{\begin{array}{c}
\Gamma=0 \\
\Omega \approx 1,24 \times 10^{9} s^{-1} \\
\chi \approx 1,11 \times 10^{12} s^{-1}
\end{array}\right.
$$




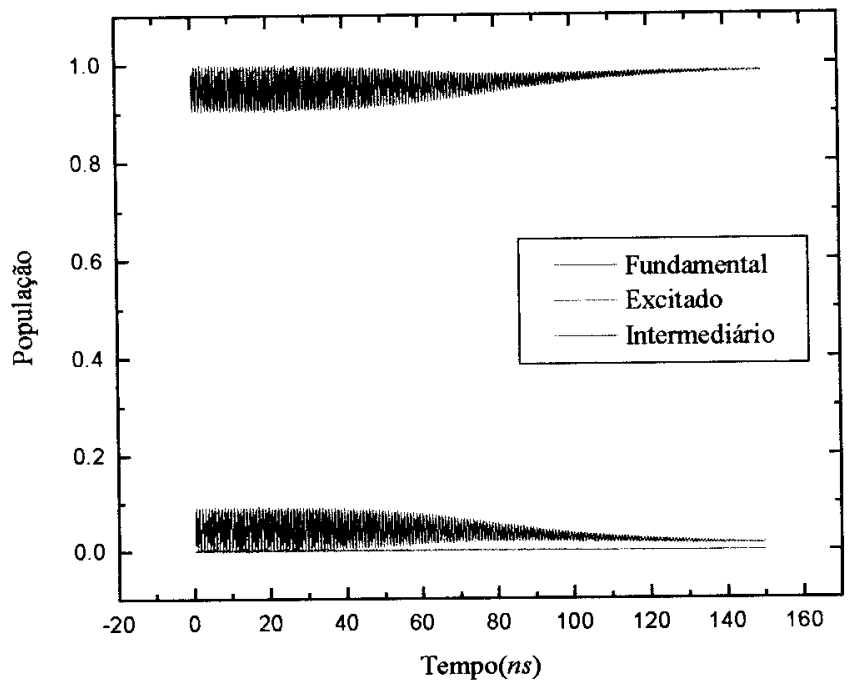

3.Gráfico da dinâmica das populações quando não há decaimento.

O gráfico acima mostra a dinâmica das populações dos estados fundamental, intermediário e excitado. Como aqui não há decaimento, as populações dos níveis fundamental e excitado oscilam devido ao acoplamento via laser de prova, e a populaçao do estado intermediário oscila com a do estado excitado devido à presença do acoplamento de estrutura fina. 


\subsection{Sistema com decaimento}

Uma vez que incluímos termos de decaimento, vamos começar nosso sistema populando apenas o excitado correspondente a $V_{e}$, de onde ocorre o decaimento. Desta forma, começamos com

$$
\begin{aligned}
& \rho_{11}(x, p, 0)=0 \\
& \rho_{22}(x, p, 0)=\rho(x, p, 0), \\
& \rho_{33}(x, p, 0)=0 .
\end{aligned}
$$

com

$$
\left\{\begin{array}{l}
\Gamma=1,25 \times 10^{7} s^{-1} \\
\Omega \approx 4,98 \times 10^{8} s^{-1} \\
\chi \approx 1,11 \times 10^{12} s^{-1}
\end{array}\right.
$$


Primeiramente, vamos fazer a análise da dinâmica da função de Wigner para o três níveis com a presença do decaimento.

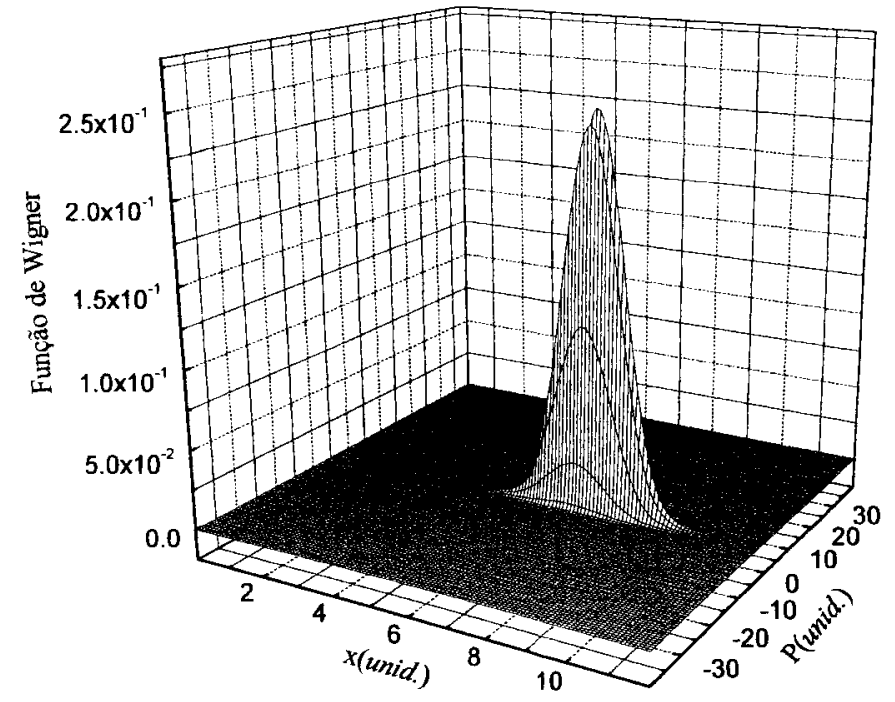

4.Função de Wigner no estado excitado após $10 n s$. 


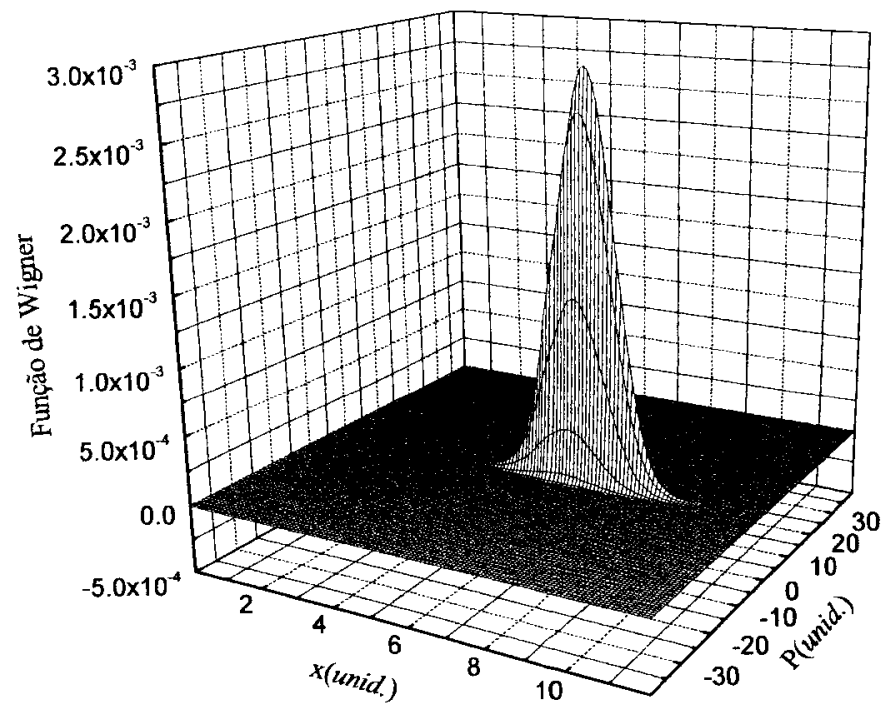

5.Função de Wigner no estado intermediário após $10 \mathrm{~ns}$. 


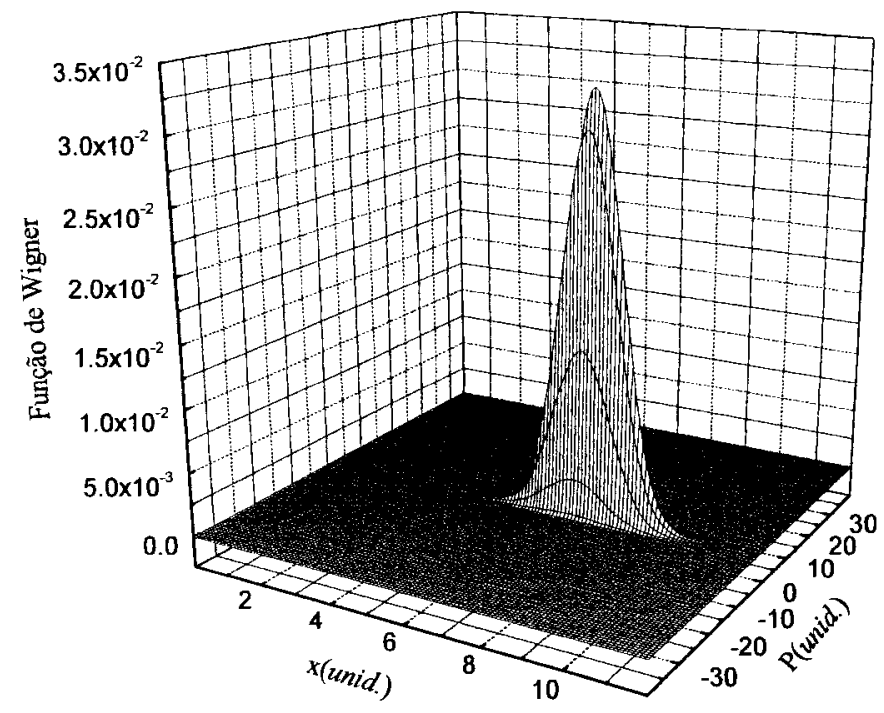

6.Função de Wigner no estado fundamental após $10 \mathrm{~ns}$.

Passados $10 n s$ vemos que tanto $\rho_{11}(x, p, 10)$, que corresponde ao estado fundamental, quanto $\rho_{33}(x, p, 10)$, que corresponde ao estado intermediário, possuem valores diferentes de zero apesar de terem iniciado o processo com população zero. Já $\rho_{22}(x, p, 10)$, que iniciou o processo com a Gaussiana da Fig. (2), já perdeu população, primeiramente para o estado excitado acoplado por $\Omega$ e posteriormente, para o intermediário acoplado por $\chi$ 
Após $60 n s$ as funções de Wigner dos três níveis estão evoluindo da seguinte forma.

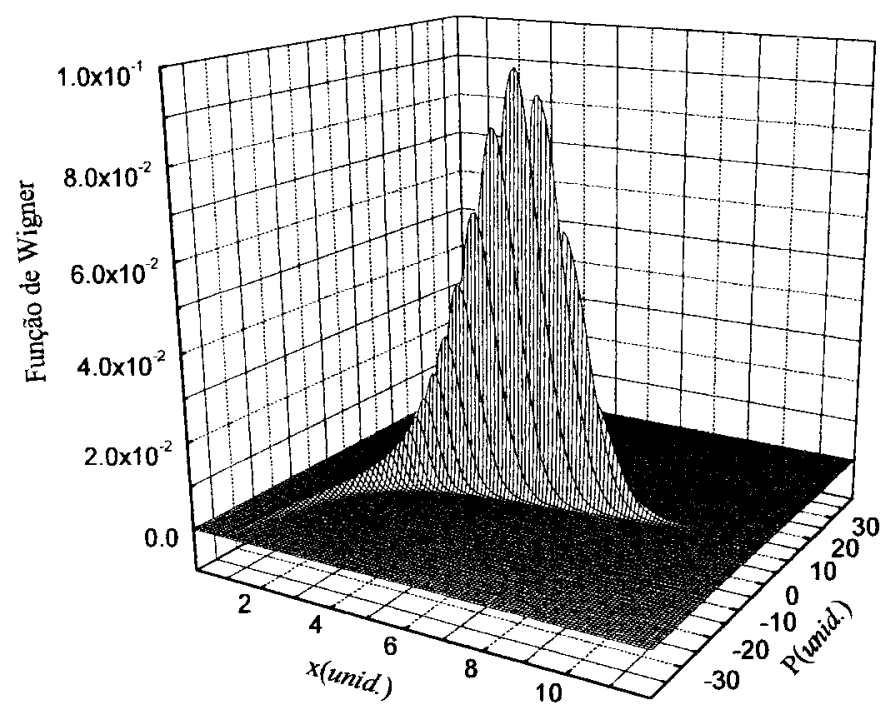

7.Função de Wigner no estado excitado após $60 \mathrm{~ns}$. 


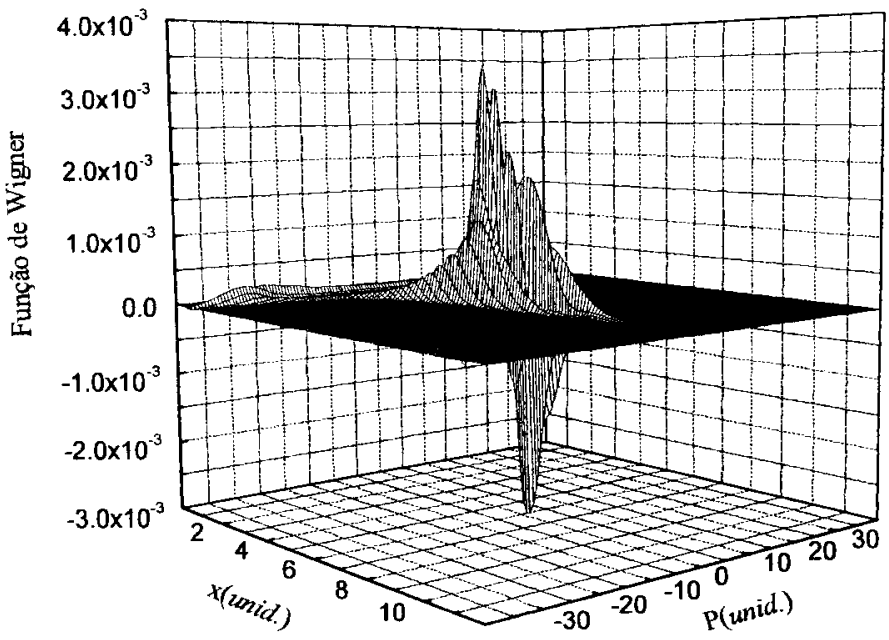

8.Função de Wigner no estado intermediário após 60 ns. 


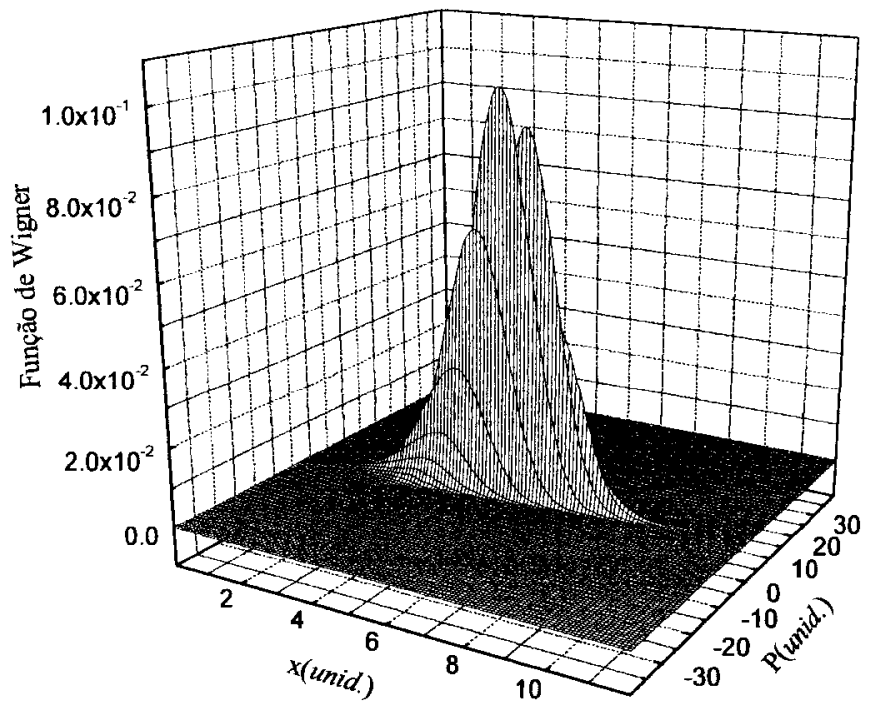

9.Função de Wigner no estado fundamental após $60 \mathrm{~ns}$.

Após $60 n s$ vemos que a população no estado fundamental já é bem maior, por conseguinte, a do estado excitado tem diminuido significativamente. As distribuições já se aproximam da origem de $x$ e começam a passar pelos cruzamentos. Note que a função de Wigner do estado intermediário asume valores negativos para determinados valores de $x \mathrm{e}$ $p$, o que é perfeitamente possível uma vez que não estamos falando de função de onda mas sim de uma função quase-probabilística que pode assumir valores negativos em estados não clássicos desde que a integral sobre $p$ e $x$, que nos fornece a probabilidade de ocupação, segundo Eq.(2.25), seja sempre positiva. Isso acontece proximo dos $60 \mathrm{~ns}$ pois a medida que a distribuição se aproxima da região do cruzamento a probabilidade de transição aumenta, cria-se, então, uma coerência o que justifica os termos negativos. 
Já no final da colisão temos:

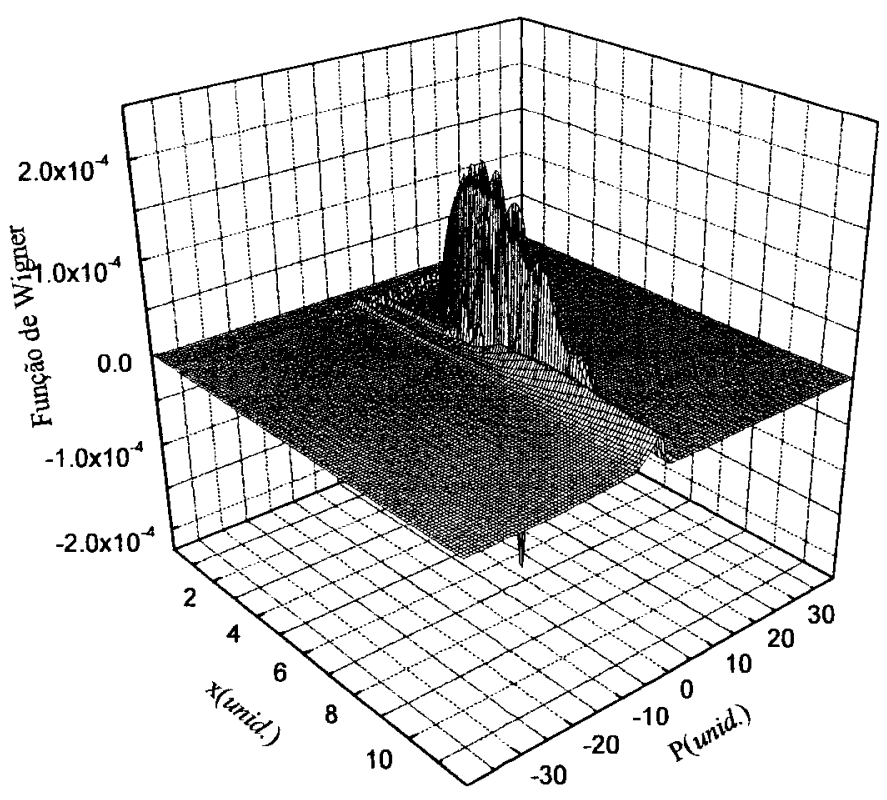

10.Função de Wigner no estado excitado após $200 \mathrm{~ns}$. 


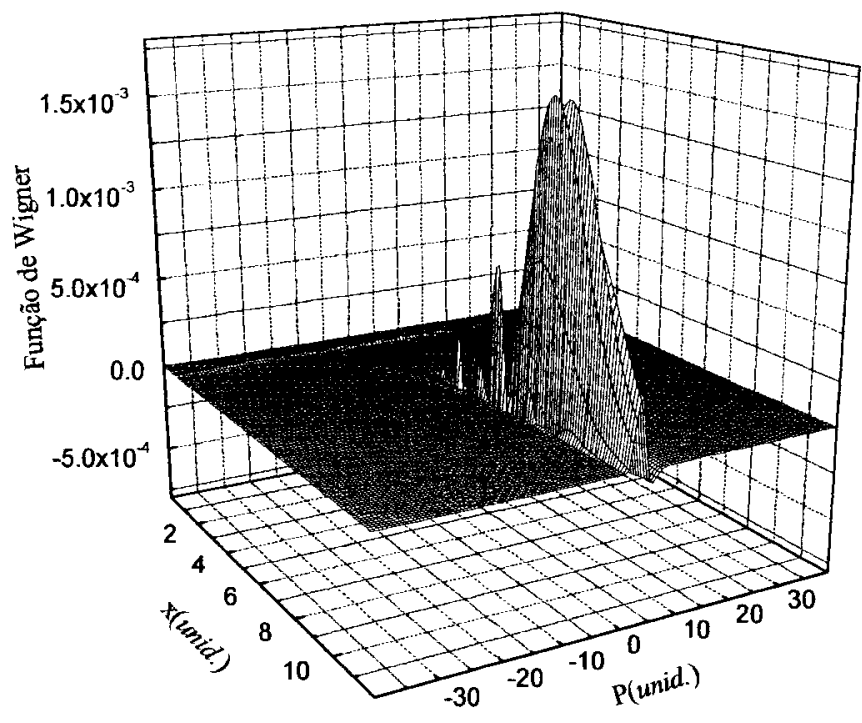

11.Função de Wigner no estado intermediário após $200 \mathrm{~ns}$. 


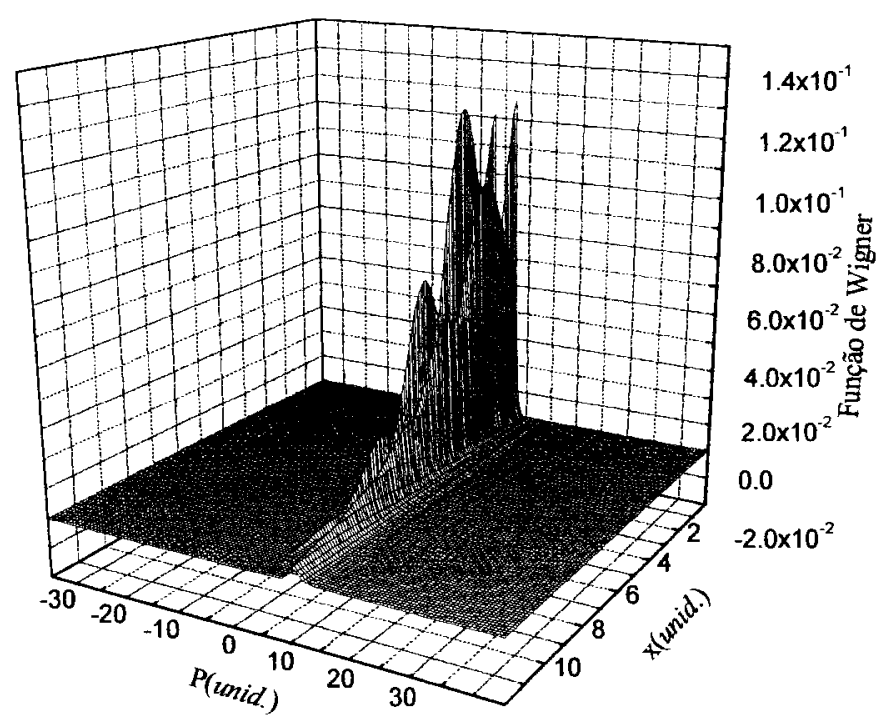

12.Função de Wigner no estado fundamental após $200 \mathrm{~ns}$.

Aqui vemos as funções de Wigner nos três estados após $200 n s$, já no final da colisão. A perda de população do estado excitado é visível. Aqui as distribuições já se afastam da origem de $x$. Todas elas já tiveram seus momenta invertidos ou pela barreira de potencial inserida por nós, ou pelo potencial repulsivo como é o caso do estado intermediário, onde há, muitas vezes a inversão de momentum antes da chegada na barreira de potencial. $O$ gráfico acima do estado fundamental ilustra muito bem, a reflexão na barreira de potencial. Note também, que novamente a função de Wigner dos estados intermediáro e excitado possuem partes negativas o que é perfertamente normal. 
Para entender melhor a dinâmica das populações veja o gráfico de população dos níveis que é apresentado a seguir.

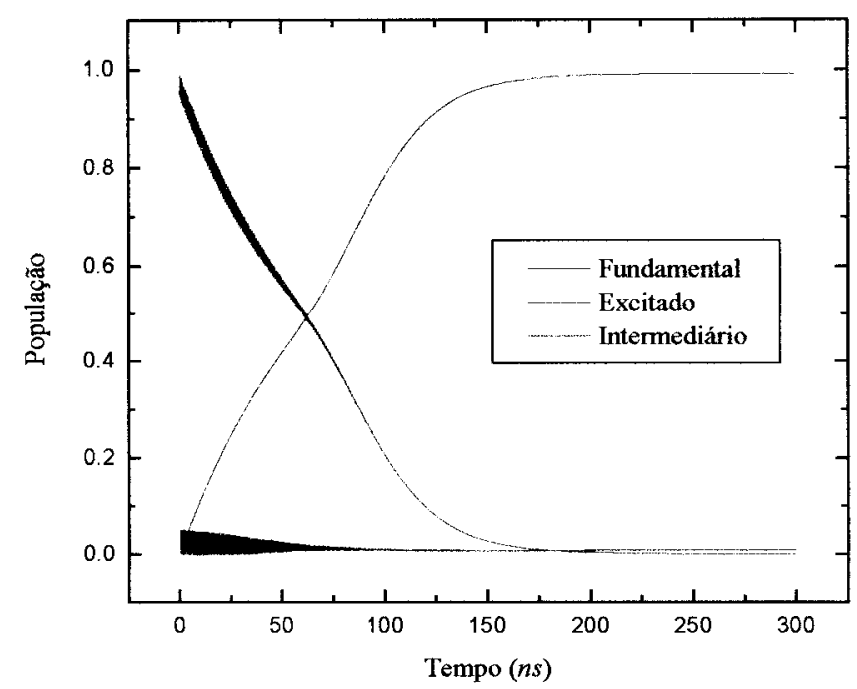

13. Gráfico da dinâmica das populações quando há decaimento.

Neste gráfico, os níveis intermediário e fundamental começam com população zero, e o nível excitado com população 1 , a população do nível excitado decai com o passar do tempo para o fundamental, e vem a se estabilizar próxima de zero. Antes disso, enquanto ainda existe população no nível excitado, notamos uma oscilação de população entre os níveis excitado e intermediário, devido ao acoplamento de estrutura fina. Assim que o nível excitado perde sua população, a população do nível intermediário pára de oscilar e também se estabiliza, mas com um valor um pouco superior. Existe também aqui o acoplamento entre o fundamental e o excitado via laser de prova, que é quase imperceptível, uma vez que tem freqüência muito menor. Com este modelo vemos que o decaimento é o processo 
dominante, sobre a mudança de estrutura fina, e deve portanto contribuir mais para as perdas em armadilhas magnéto-ópticas. 
Para entender melhor como os processos colisionais, de escape radiativo e mudança de estrutura fina contribuem para o aumento da energia cinética dos átomos em colisão e por conseguinte às perdas em armadilhas, vamos examinar os gráficos a seguir que mostram a distribuição de momemtum antes e depois da colisão.

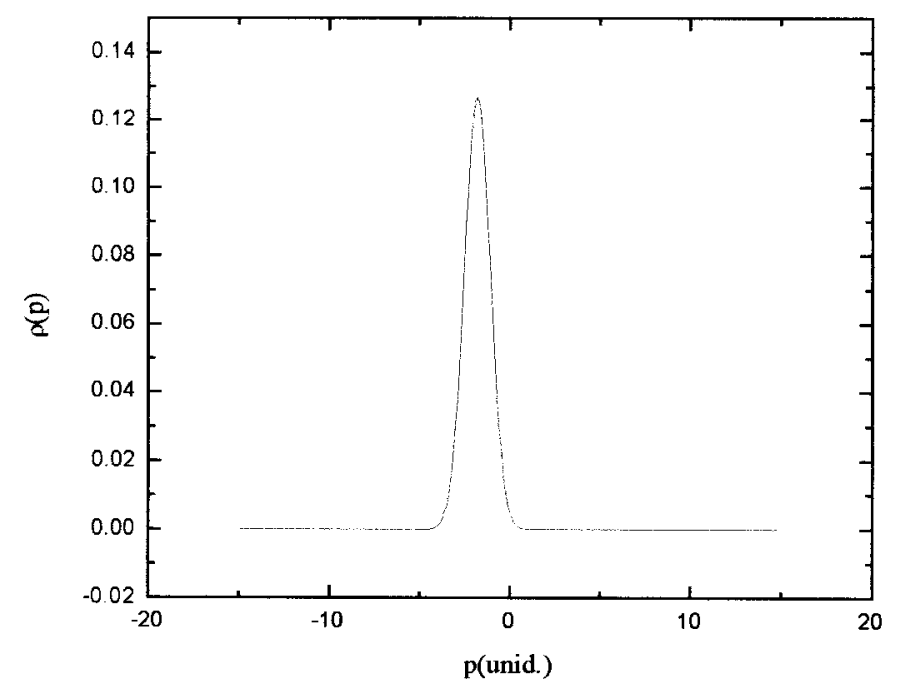

14.Gráfico da distribuição de momentum no estado fundamental antes da colisão.

O gráfico acima mostra a distribuição de momentum no estado fundamental antes que a colisão ocorra. Note que a curva está distribuída em momenta de módulos entre 0 e 5 (unid.). Vemos também que $\rho(p)$ tem valores menores que no gráfico a seguir; isto porque este nível começou com população zero e vem ganhando população por causa do decaimento. 


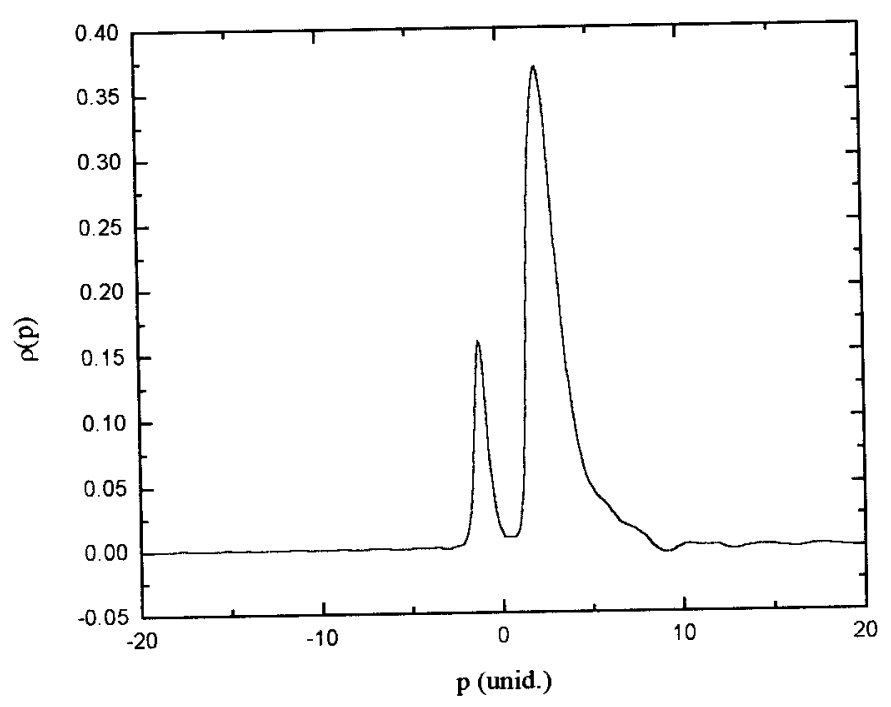

15.Gráfico da distribuição de momentum no estado fundamental após a colisão.

O Gráfico acima mostra a distribuição de momentum no estado fundamental após a colisão. Note que existe uma certa quantidade de átomos com momentum de módulo maior que 5 (unid.), o que não existia antes da colisão, mostrando assim que alguns átomos tiveram tempo suficiente de ganhar energia cinética no potencial excitado (que é atrativo), antes de decair. Estes átomos decaíram e chegaram, então, ao potencial fundamental com energia cinética superior, o que pode causar a perda conhecida como escape radiativo. 


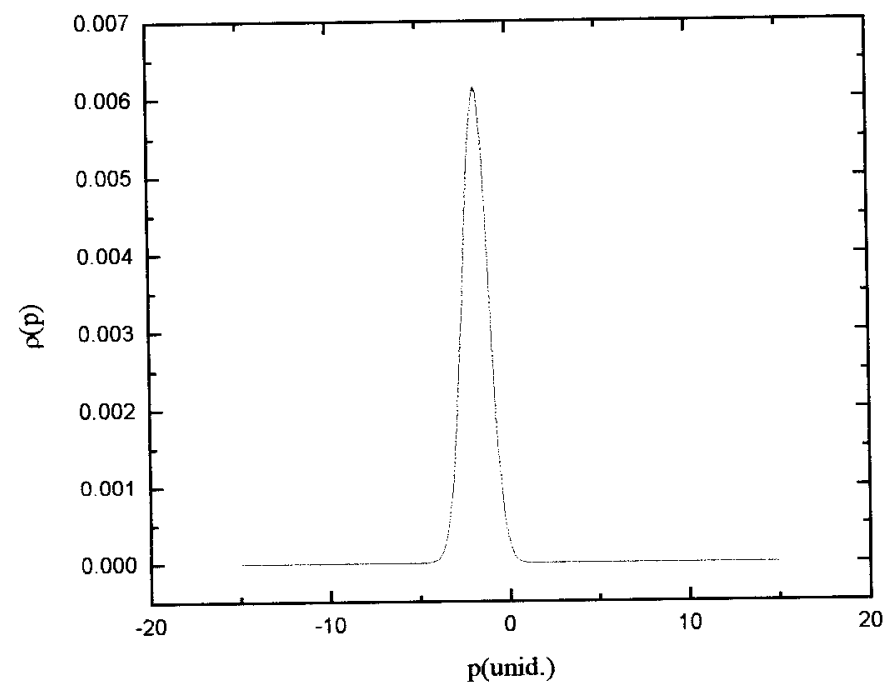

16.Gráfico da distribuição de momentum no estado intermediário antes da colisão.

O gráfico acima mostra a distribuição de momentum antes da colisão no nível intermediário. Novamente, vemos os valores distribuídos em momenta de módulo entre 0 e 5 (unid.). 


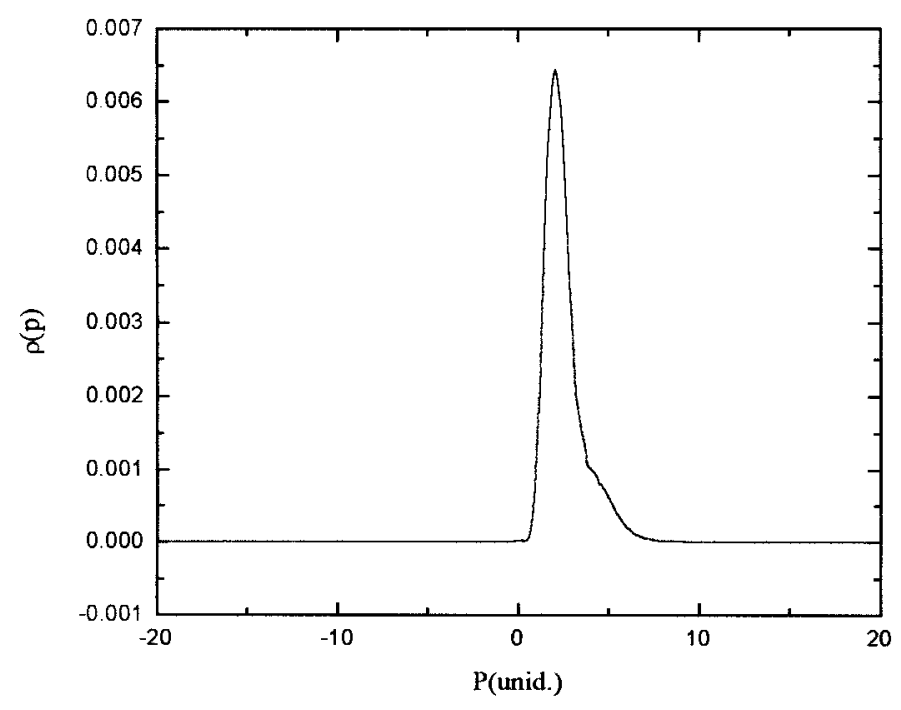

17.Gráfico da distribuição de momentum para o estado intermediário após a colisão.

O Gráfico acima mostra a distribuição de momentum para o estado intermediário após a colisão. Assim como no caso anterior existe uma quantidade de átomos com momentum de módulo superior ao que possuíam antes da colisão. Se estes átomos tiverem energia suficiente para escapar da armadilha, esta perda é denominada de perda por mudança de estrutura fina. Note que a ordem de grandeza desta distribuição é menor do que a da distribuição no estado fundamental. Isto mostra que menos átomos envolvidos neste processo devem escapar se comparados com a quantidade dos mesmos que deve escapar via escape radiativo.

Devemos ressaltar que em nenhum dos dois casos o acréscimo de energia foi sulficiente para causar o escape de uma armadilha magneto-óptica real, que tem profundidade típica da ordem de $1 K$, o que exigiria um momentum da ordem de 114 (unid.). No entanto, é possível notar aqui o mesmo comportamento físico que é esperado quando trata- 
mos os processos colisionais de átomos frios. É importante ressaltar também que este é um modelo simples e até mesmo ingênuo no tratamento de tais processos, mas uma vez desenvolvido tal método e implementado poderemos, no futuro, sofisticar o modelo levando em conta parâmetros que aqui, nesta primeira abordagem, foram desprezados, e assim, chegar o mais próximo possível dos resultados obtidos em laboratório.

Podemos, então, calcular a fração de átomos que escapa de uma armadilha magnetoóptica.

$$
\begin{aligned}
\frac{\Delta N}{N} & =\int_{P_{c}}^{\infty} d p\left(\left|\phi_{1}(p, \infty)\right|^{2}+\left|\phi_{2}(p, \infty)\right|^{2}+\left|\phi_{3}(p, \infty)\right|^{2}\right) \\
& =\int_{P_{c}}^{\infty} d p \int_{-\infty}^{\infty} d x W(x, p, \infty) .
\end{aligned}
$$

onde $P c$ é o momentum crítico para escape da armadilha, ou seja, o valor mínimo de momentum que o átomo tem que ter para escapar da armadilha magneto-óptica. 
Se aumentarmos o decaimento em 10 vezes, teremos o seguinte gráfico

$$
\left\{\begin{array}{l}
\Gamma=1,25 \times 10^{8} s^{-1} \\
\Omega \approx 4,98 \times 10^{8} s^{-1} \\
\chi \approx 1,11 \times 10^{12} s^{-1}
\end{array}\right.
$$

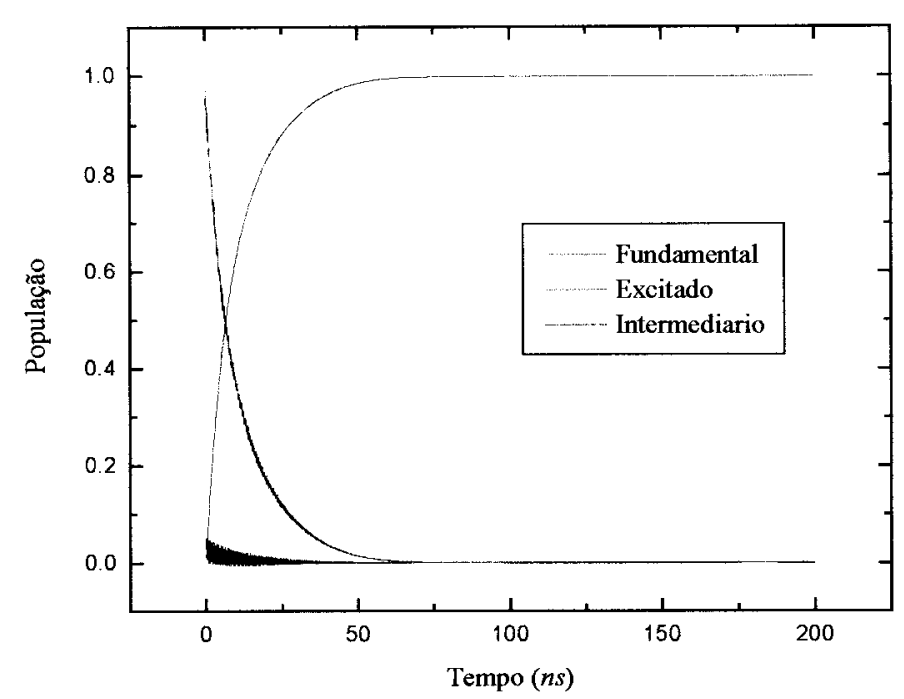

18.Gráfico de população durante a colisão com decaimento 10 vezes maior.

Vemos aqui, como era de se esperar com o aumento da taxa de decaimento, que a troca de população entre os níveis excitado e fundamental, se dá ainda mais rapidamente. Podemos notar que a oscilação de população entre os níveis excitado e intermediário é ainda menor e como o nível excitado perde população muito rapidamente, não há tempo para que a mudança de estrutura fina ocorra e por isso o nível intermediário fica ainda menos populado. 


\section{Capítulo 5: Conclusões}

\subsection{Contribuições originais deste trabalho}

- Neste trabalho obtivemos os primeiros resultados para colisões frias utilizando o formalismo de Wigner.

- O algoritmo inédito, desenvolvido e implementado por nós, poderá ser utilizado para cálculos futuros de modelos colisionais mais sofisticados.

- Os resultados obtidos aqui com este algorítmo mostram que os processos colisionais são mesmo fenômenos dissipativos.

\subsection{Conclusões importantes a respeito do modelo}

Desenvolvemos, utilizando o formalismo de Wigner, um conjunto de equações diferenciais inéditas no tratamento de colisões frias. Resolvemos estas equações numéricamente, fazendo uso de um algoritmo, também inédito, desenvolvido por nós durante o desenrolar deste trabalho. $\mathrm{O}$ algoritmo se mostrou eficiente para o cálculo de sistemas deste gênero. É importante dizer que este algoritmo também foi exaustivamente testado para sistemas os quais a solução analítica é conhecida e teve resultados compatíveis. 
Apresentamos, então, alguns dos resultados obtidos com este algoritmo para o sistema de três níveis proposto no Capítulo 2. Primeiramente, sem colocar os termos de decaimento analisamos a dinâmica das populações dos três níveis.

Em seguida, utilizando os termos de decaimento, fizemos a análise da dinâmica colisional, observando os gráficos da função de Wigner no espaço de fase para diferentes tempos de colisão. Também analisamos, neste caso, a dinâmica de populações dos três níveis.

Por fim, fizemos a análise das distribuições de momentum antes e depois da colisão. Vimos que tanto o processo de mudança de estrutura fina como o processo de escape radiativo proporcionam um aumento da energia cinética o que, em muitos, casos pode causar a perda de átomos em uma nuvem confinada.

\subsection{Sugestões para trabalhos complementares}

Agora que o programa foi implementado com sucesso poderemos no futuro, como um possível doutorado, generalizar os cálculos para o caso tridimensional, isto é, incluindo os graus de liberdade de rotação da "quase-molécula". Comparar os resultados com as previsões da teoria de Landau-Zener e com as previsões baseadas na matriz de espalhamento obtida pela resolução da equação matricial de Schrödinger correspondente, e até, incluir correções quânticas para o movimento traslacional, incluindo termos de ordens maiores de $\hbar$ na expansão de $J_{m n}$. 


\section{Referências}

[1] S. Chu, Rev. Mod. Phys. 70, 685 (1998); C. N. Cohen-Tannoudji, Rev. Mod. Phys. 70, 707 (1998); W. D. Phillips, Rev. Mod. Phys. 70, 721 (1998).

[2] J. Weiner, V. S. Bagnato, S. C. Zilio e P. S. Julienne; Rev. Mod. Phys. 71, 1 (1999)

[3] E. Wigner, Phys. Rev. A 40, 749 (1932).

[4] M. Hillery, R. F. O’ Connell, M. O. Scully, E. P. Wigner, Physics Reports, 106, 121, (1984)

[5] M. A. Marchiolli, "A Formulação de Weyl-Wigner da Mecânica Quântica no Espaço de Fase" (Apostila do curso de verão do IFSC, 2002).

[6] R. J. Cook, Phys. Rev. A 22, 1078 (1980).

[7] K.-A. Suominen, Y. B. Band, I. Tuvi, K. Burnett e P. S. Julienne, Phys. Rev. A 57, 3724 (1998).

[8] S. Groot, "La Transformation de Weyl et la Fonction de Wigner: Une Forme Alternative de la Mécanique Quantique" (Les presses de l'Université de Montréal, 1974).

[9] L. Allen e J. H. Eberly, "Optical Ressonance and Two- Level Atoms" (Wiley, N. Y., 1975)

[10] W. H. Press, S. A. Tteukolsky, W. T. Vetterling, B. P. Flannery, "Numerical Recipes in FORTRAN" (Cambridge University Press).

[11] S. Stenholm, Phys. Rev. A 47, 2523 (1993). 


\section{Apêndice A: Cálculo do Hamiltoniano}

A interação do laser com os átomos, desprezando os efeitos de transições proibidas pelas regras de seleção de dipolo, é:

$$
H_{L-A}=-\vec{d}_{1} \cdot \vec{E}_{p r}\left(\vec{R}_{1}, t\right)-\vec{d}_{2} \cdot \vec{E}_{p r}\left(\vec{R}_{2}, t\right)
$$

onde $\vec{d}_{1}$ é o operador de dipolo do átomo 1 e $\vec{d}_{2}$ é referente ao átomo $2 ; \vec{R}_{1} \mathrm{e} \vec{R}_{2}$ são os operadores posição dos átomos 1 e 2 , respectivamente.

Em todos os trabalhos desta área, para simplificar o tratamento, considera-se apenas uma polarização do campo elétrico presente e escolhe-se a amplitude $E_{q}(\mathbf{R})$ como a de apenas um feixe laser propagando-se em uma única direção e aproximado por uma onda plana, $E_{q}(\mathbf{R})=E_{c} \exp \left[i \mathbf{k}_{L} \cdot \mathbf{R}\right]$, com $E_{c}$ constante e $\mathbf{k}_{L}$ o vetor de onda do feixe. $O$ índice $q$ corresponde à polarização descrita pelo versor $\hat{\mathbf{e}}_{q}=\hat{\mathbf{e}}_{q}\left(\hat{\mathbf{k}}_{L}\right)$, que pode ser $q= \pm 1$ para polarização circular e $q=0$ para linear; $\hat{\mathbf{e}}_{q}=\hat{\mathbf{e}}_{q}\left(\hat{\mathbf{k}}_{L}\right)$ é definido em relação ao eixo de propagação $\hat{\mathbf{k}}_{L}\left(\hat{\mathbf{e}}_{0}\right.$ é tomado perpendicularmente a $\left.\hat{\mathbf{k}}_{L}\right)$. Este seria o caso de um experimento real em que se desligam os feixes aprisionantes e introduz-se um feixe laser de prova da forma

$$
\vec{E}_{p r}(\vec{R}, t)=\frac{E_{c}}{2} \widehat{e}_{q} \exp \left(i \vec{k}_{L} \cdot \vec{R}-i \omega_{L} t\right)+\frac{E_{c}^{*}}{2} \widehat{e}_{q}^{*} \exp \left(-i \vec{k}_{L} \cdot \vec{R}+i \omega_{L} t\right)
$$

Portanto, 


$$
\begin{aligned}
H_{L-A}= & -\frac{E_{c}}{2} \exp \left(-i \omega_{L} t\right) \widehat{e}_{q} \cdot \vec{d}_{1} \exp \left(i \vec{k}_{L} \cdot \vec{R}_{1}\right) \\
& -\frac{E_{c}}{2} \exp \left(-i \omega_{L} t\right) \widehat{e}_{q} \cdot \vec{d}_{2} \exp \left(i \vec{k}_{L} \cdot \vec{R}_{2}\right) \\
& -\frac{E_{c}^{*}}{2} \exp \left(i \omega_{L} t\right) \widehat{e}_{q}^{*} \cdot \vec{d}_{1} \exp \left(-i \vec{k}_{L} \cdot \vec{R}_{1}\right) \\
& -\frac{E_{c}^{*}}{2} \exp \left(i \omega_{L} t\right) \widehat{e}_{q}^{*} \cdot \vec{d}_{2} \exp \left(-i \vec{k}_{L} \cdot \vec{R}_{2}\right)
\end{aligned}
$$

Aqui supomos que o campo elétrico varia muito pouco na região do orbital eletrônico, de forma que podemos calcular $\vec{E}_{p r}$ sobre o operador posição de cada centro de massa atômico e não sobre a posição de cada elétron sem maiores transtornos.

Assim,

$$
\begin{aligned}
& H_{L-A}=-\frac{E_{c}}{2} \exp \left(i \vec{k}_{L} \cdot \vec{R}_{C M}-i \omega_{L} t\right) \widehat{e}_{q} \cdot \vec{d}_{1} \exp \left[\frac{i \vec{k}_{L}}{2} \cdot\left(\vec{R}_{1}-\vec{R}_{2}\right)\right] \\
& -\frac{E_{c}}{2} \exp \left(i \vec{k}_{L} \cdot \vec{R}_{C M}-i \omega_{L} t\right) \widehat{e}_{q} \cdot \vec{d}_{2} \exp \left[\frac{i \vec{k}_{L}}{2} \cdot\left(\vec{R}_{2}-\vec{R}_{1}\right)\right] \\
& -\frac{E_{c}^{*}}{2} \exp \left(i \vec{k}_{L} \cdot \vec{R}_{C M}+i \omega_{L} t\right) \widehat{e}_{q}^{*} \cdot \vec{d}_{1} \exp \left[\frac{i \vec{k}_{L}}{2} \cdot\left(\vec{R}_{2}-\vec{R}_{1}\right)\right] \\
& -\frac{E_{c}^{*}}{2} \exp \left(i \vec{k}_{L} \cdot \vec{R}_{C M}+i \omega_{L} t\right) \widehat{e}_{q}^{*} \cdot \vec{d}_{2} \exp \left[\frac{i \vec{k}_{L}}{2} \cdot\left(\vec{R}_{1}-\vec{R}_{2}\right)\right] \text {, }
\end{aligned}
$$

$$
\begin{aligned}
H_{L-A}= & -\frac{E_{c}}{2} \exp \left(i \vec{k}_{L} \cdot \vec{R}_{C M}-i \omega_{L} t\right) \widehat{e}_{q} \cdot \vec{d}_{1} \exp \left(-\frac{i \vec{k}_{L}}{2} \cdot \vec{r}\right) \\
& -\frac{E_{c}}{2} \exp \left(i \vec{k}_{L} \cdot \vec{R}_{C M}-i \omega_{L} t\right) \widehat{e}_{q} \cdot \vec{d}_{2} \exp \left(\frac{i \vec{k}_{L}}{2} \cdot \vec{r}\right)
\end{aligned}
$$




$$
\begin{aligned}
& -\frac{E_{c}^{*}}{2} \exp \left(i \vec{k}_{L} \cdot \vec{R}_{C M}+i \omega_{L} t\right) \widehat{e}_{q}^{*} \cdot \vec{d}_{1} \exp \left(\frac{i \vec{k}_{L}}{2} \cdot \vec{r}\right) \\
& -\frac{E_{c}^{*}}{2} \exp \left(i \vec{k}_{L} \cdot \vec{R}_{C M}+i \omega_{L} t\right) \widehat{e}_{q}^{*} \cdot \vec{d}_{2} \exp \left(-\frac{i \vec{k}_{L}}{2} \cdot \vec{r}\right) .
\end{aligned}
$$

A interação átomo-átomo é dada por

$$
\begin{aligned}
\hat{H}_{A-A}= & V_{1}(|\vec{r}|)|1\rangle\left\langle 1\left|+V_{2}(|\vec{r}|)\right| 2\right\rangle\langle 2| \\
& +V_{3}(|\vec{r}|)|3\rangle\langle 3|+\hbar \varkappa(|3\rangle\langle 2|+| 2\rangle\langle 3|),
\end{aligned}
$$

onde $V_{1}(|\vec{r}|)$ é a interação correlacionando-se, assintoticamente, com ambos os átomos em seus estados ${ }^{2} S_{1 / 2} ; V_{2}(|\vec{r}|)$ correlacionando-se com ${ }^{2} S_{1 / 2}+{ }^{2} P_{3 / 2} ; V_{3}(|\vec{r}|)$ com ${ }^{2} S_{1 / 2}+{ }^{2} P_{1 / 2}$ e, por fim, $\hbar \varkappa$ é igual à separação entre as energias dos termos ${ }^{2} P_{3 / 2}$ e ${ }^{2} P_{1 / 2}$ devida à interação spin-órbita.

Assim, o Hamiltoniano total é:

$$
\hat{H}=\frac{\vec{P}_{C M}^{2}}{2 M}+\frac{\vec{p}^{2}}{2 \mu}+H_{A-A}+H_{L-A}
$$

onde $\mu$ é a massa reduzida de dois átomos idênticos, $\vec{p}$ é o momenum relativo e $M=2 m$ é a massa da "quase-molécula".

Agora algumas aproximações devem ser levadas em conta. A título de simplificação faze$\operatorname{mos} \exp \left( \pm \frac{i \vec{k}_{L}}{2} \cdot \vec{r}\right) \approx 1$ na equação (6.56). Ainda na mesma equação, desprezamos o termo $\exp \left( \pm i \vec{k}_{L} \cdot \vec{R}_{C M}\right)$, pois tomamos $\vec{R}_{C M}\left|\Psi_{0}\right\rangle=0$, no referencial do centro de massa, para o instante inicial, e supomos que este auto-estado seja estacionário durante o espalhamento, o que é equivalente a fazer a aproximação de que o centro de massa 
da "quase-molécula" sofra um deslocamento lento comparado com a escala de tempo da dinâmica colisional relativa. Esta hipótese nos leva à aproximação $\frac{P_{C M}^{2}}{2 M}=0$. Escrevendo o Hamiltoniano total, utilizando as equações (6.56) e (6.57) com as devidas aproximações, obtemos:

$$
\begin{aligned}
\hat{H}= & \frac{p^{2}}{2 \mu}+V_{1}(|\vec{r}|)|1\rangle\left\langle 1\left|+V_{2}(|\vec{r}|)\right| 2\right\rangle\langle 2| \\
& +V_{3}(|\vec{r}|)|3\rangle\langle 3|+\hbar \varkappa(|3\rangle\langle 2|+| 2\rangle\langle 3|) \\
& -\frac{E_{c}}{2} \exp \left(-i \omega_{L} t\right) \widehat{e}_{q} \cdot \vec{d}_{M}-\frac{E_{c}^{*}}{2} \exp \left(i \omega_{L} t\right) \widehat{e}_{q}^{*} \cdot \vec{d}_{M}
\end{aligned}
$$

onde $\vec{d}_{M}=\vec{d}_{1}+\vec{d}_{2}=\vec{D}_{M}|1\rangle\left\langle 2\left|+\vec{D}_{M}^{*}\right| 2\right\rangle\langle 1| \mathrm{e} \vec{D}_{M}=\left\langle 1\left|\vec{d}_{M}\right| 2\right\rangle$. Podemos, ainda, reescrever a equação acima, definindo a frequência de Rabi $\Omega=\frac{E_{c}\left(\widehat{e}_{q} \cdot \vec{D}_{M}\right)}{2 \hbar}=\Omega^{*} \mathrm{e}$ os operadores $\hat{s}=|1\rangle\left\langle 2\left|, \hat{s}^{\dagger}=\right| 2\right\rangle\langle 1|, \hat{\sigma}=| 2\rangle\langle 3|$ e $\hat{\sigma}^{\dagger}=|3\rangle\langle 2|$ dos estados internos da "quase-molécula":

$$
\begin{aligned}
\hat{H}= & \frac{p^{2}}{2 \mu}+\left[V_{1}(r)\right] \hat{s} \hat{s}^{\dagger}+\left[V_{2}(r)\right] \hat{s}^{\dagger} \hat{s}+\left[V_{3}(r)\right] \hat{\sigma}^{\dagger} \hat{\sigma} \\
& -\hbar \Omega\left\{\left[\exp \left(-i \omega_{L} t\right)\right] \hat{s}^{\dagger}+\left[\exp \left(i \omega_{L} t\right)\right] \hat{s}\right\}+\frac{\hbar \varkappa}{2}\left(\hat{\sigma}^{\dagger}+\hat{\sigma}\right) .
\end{aligned}
$$




\section{Apêndice B: Cálculo das Equações Diferenciais}

Podemos escrever nosso Hamiltoniano em termos do operador de Wigner,

$$
\begin{aligned}
\widehat{H}= & \frac{\widehat{p}_{x}^{2}}{2 \mu}+\iint d x d p\left[V_{1}(x)\right] \widehat{f}(x, p)|1\rangle\langle 1|+ \\
& +\iint d x d p\left[V_{2}(x)\right] \widehat{f}(x, p)|2\rangle\left\langle 2\left|+\iint d x d p\left[V_{3}(x)\right] \widehat{f}(x, p)\right| 3\right\rangle\langle 3|- \\
& -\hbar \Omega\left\{\left[\exp \left(-i \omega_{L} t\right)\right]|2\rangle\left\langle 1\left|+\left[\exp \left(i \omega_{L} t\right)\right]\right| 1\right\rangle\langle 2|\right\}+\frac{\hbar \varkappa}{2}(|3\rangle\langle 2|+| 2\rangle\langle 3|) .
\end{aligned}
$$

Fazendo a evolução temporal do operador de Wigner, segundo a equação de Heisenberg,

$$
\frac{\partial \widehat{f}(x, p)}{\partial t}=\frac{1}{i \hbar}[\widehat{f}(x, p), \widehat{H}]
$$

teremos

$$
\begin{aligned}
\frac{\partial \widehat{f}(x, p)}{\partial t}= & \frac{1}{i \hbar}\left[\widehat{f}(x, p), \frac{\widehat{p}_{x}^{2}}{2 \mu}\right]+\frac{1}{i \hbar}\left[\widehat{f}(x, p), \iint d x d p\left[V_{1}(x)\right] \widehat{f}(x, p)|1\rangle\langle 1|\right]+ \\
& +\frac{1}{i \hbar}\left[\widehat{f}(x, p), \iint d x d p\left[V_{2}(x)\right] \widehat{f}(x, p)|2\rangle\langle 2|\right]+ \\
& +\frac{1}{i \hbar}\left[\widehat{f}(x, p), \iint d x d p\left[V_{3}(x)\right] \widehat{f}(x, p)|3\rangle\langle 3|\right]+ \\
& +\frac{1}{i \hbar}\left[\widehat{f}(x, p),-\hbar \Omega\left\{\left[\exp \left(-i \omega_{L} t\right)\right]|2\rangle\left\langle 1\left|+\left[\exp \left(i \omega_{L} t\right)\right]\right| 1\right\rangle\langle 2|\right\}\right]+ \\
& +\frac{1}{i \hbar}\left[\widehat{f}(x, p), \frac{\hbar \varkappa}{2}(|3\rangle\langle 2|+| 2\rangle\langle 3|)\right]
\end{aligned}
$$

o que, calculando termo a termo, é 


$$
\begin{gathered}
\frac{1}{i \hbar}\left[\widehat{f}(x, p), \frac{\hat{p}_{x}^{2}}{2 \mu}\right]=\iint d x^{\prime} d p^{\prime} \frac{\hat{p}_{x}^{2}}{2 \mu}\left[\widehat{f}(x, p), \widehat{f}\left(x^{\prime}, p^{\prime}\right)\right] \\
=-\frac{p}{\mu} \frac{\partial}{\partial x} \widehat{f}(x, p), \\
\frac{1}{i \hbar}\left[\widehat{f}(x, p), \iint d x d p\left[V_{1}(x)\right] \widehat{f}(x, p)|1\rangle\langle 1|\right]=|1\rangle\langle 1| \int d p^{\prime} J_{11}\left(x, p-p^{\prime}\right) \widehat{f}\left(x, p^{\prime}\right), \\
\frac{1}{i \hbar}\left[\widehat{f}(x, p), \iint d x d p\left[V_{2}(x)\right] \widehat{f}(x, p)|2\rangle\langle 2|\right]=|2\rangle\langle 2| \int d p^{\prime} J_{22}\left(x, p-p^{\prime}\right) \widehat{f}\left(x, p^{\prime}\right), \\
\frac{1}{i \hbar}\left[\widehat{f}(x, p), \iint d x d p\left[V_{3}(x)\right] \widehat{f}(x, p)|3\rangle\langle 3|\right]=|3\rangle\langle 3| \int d p^{\prime} J_{33}\left(x, p-p^{\prime}\right) \widehat{f}\left(x, p^{\prime}\right), \\
\left.\cdot \int \widehat{f}(x, p),-\hbar \Omega\left\{\left[\exp \left(-i \omega_{L} t\right)\right]|2\rangle\left\langle 1\left|+\left[\exp \left(i \omega_{L} t\right)\right]\right| 1\right\rangle\langle 2|\right\}\right]=0, \\
\frac{1}{i \hbar}\left[\widehat{f}(x, p), \frac{\hbar \varkappa}{2}(|3\rangle\langle 2|+| 2\rangle\langle 3|)\right]=0 .
\end{gathered}
$$

Vemos, aqui, que o resultado dependerá da evolução dos operadores $|1\rangle\langle 1| \widehat{f}(x, p)$,

$|2\rangle\langle 2| \widehat{f}(x, p)$ e $|3\rangle\langle 3| \widehat{f}(x, p)$ e estes, por sua vez, dependerão de $|1\rangle\langle 2| \widehat{f}(x, p)$,

$|2\rangle\langle 3| \widehat{f}(x, p)$ e $|3\rangle\langle 1| \widehat{f}(x, p)$, e assim sucessivamente, até obtermos o conjunto de equações acopladas:

$$
\begin{aligned}
\left(\frac{\partial}{\partial t}+\frac{p}{\mu} \frac{\partial}{\partial x}\right) \widehat{f}(x, p)= & \int d p^{\prime}|1\rangle\langle 1| J_{11}\left(x, p-p^{\prime}\right) \widehat{f}\left(x, p^{\prime}\right)+ \\
& +\int d p^{\prime}|2\rangle\langle 2| J_{22}\left(x, p-p^{\prime}\right) \widehat{f}\left(x, p^{\prime}\right)+ \\
& +\int d p^{\prime}|3\rangle\langle 3| J_{33}\left(x, p-p^{\prime}\right) \widehat{f}\left(x, p^{\prime}\right)
\end{aligned}
$$




$$
\begin{aligned}
\left(\frac{\partial}{\partial t}+\frac{p}{\mu} \frac{\partial}{\partial x}\right)[|1\rangle\langle 1| \widehat{f}(x, p)]= & |1\rangle\langle 1| \int d p^{\prime} J_{11}\left(x, p-p^{\prime}\right) \widehat{f}\left(x, p^{\prime}\right)+ \\
& +i \Omega\left[-\exp \left(-i \omega_{L} t\right)\right]|2\rangle\langle 1| \widehat{f}(x, p)+ \\
& +i \Omega\left[\exp \left(i \omega_{L} t\right)\right]|1\rangle\langle 2| \widehat{f}(x, p) .
\end{aligned}
$$

De maneira análoga calculamos as equações:

$$
\begin{aligned}
\left(\frac{\partial}{\partial t}+\frac{p}{\mu} \frac{\partial}{\partial x}\right)[|2\rangle\langle 2| \widehat{f}(x, p)]= & |2\rangle\langle 2| \int d p^{\prime} J_{22}\left(x, p-p^{\prime}\right) \widehat{f}\left(x, p^{\prime}\right)+ \\
& +i \Omega\left[\exp \left(-i \omega_{L} t\right)\right]|2\rangle\langle 1| \widehat{f}(x, p)- \\
& -i \Omega\left[\exp \left(i \omega_{L} t\right)\right]|1\rangle\langle 2| \widehat{f}(x, p)+ \\
& +\frac{i \varkappa}{2}(|3\rangle\langle 2|-| 2\rangle\langle 3|) \widehat{f}(x, p), \\
\left(\frac{\partial}{\partial t}+\frac{p}{\mu} \frac{\partial}{\partial x}\right)[|3\rangle\langle 3| \widehat{f}(x, p)]= & |3\rangle\langle 3| \int d p^{\prime} J_{33}\left(x, p-p^{\prime}\right) \widehat{f}\left(x, p^{\prime}\right)+ \\
& +\frac{i \varkappa}{2}(|2\rangle\langle 3|-| 3\rangle\langle 2|) \widehat{f}(x, p) .
\end{aligned}
$$

Note que, nas Equações (7.63), (7.64) e (7.65) aparecem os operadores $|1\rangle\langle 2| \widehat{f}(x, p)$, $|2\rangle\langle 1|\widehat{f}(x, p)| 2\rangle,\langle 3| \widehat{f}(x, p)$ e $|3\rangle\langle 2| \widehat{f}(x, p)$, o que nos leva a calcular as equações subseqüentes:

$$
\begin{aligned}
\left(\frac{\partial}{\partial t}+\frac{p}{\mu} \frac{\partial}{\partial x}\right)[|1\rangle\langle 2| \widehat{f}(x, p)]= & |1\rangle\langle 2| \int d p^{\prime} J_{21}\left(x, p^{\prime}-p\right) \widehat{f}\left(x, p^{\prime}\right)+ \\
& +i \Omega \exp \left(-i \omega_{L} t\right)|1\rangle\langle 1| \widehat{f}(x, p)- \\
& -i \Omega \exp \left(-i \omega_{L} t\right)|2\rangle\langle 2| \widehat{f}(x, p)+
\end{aligned}
$$




$$
-\frac{i \varkappa}{2}|1\rangle\langle 3| \widehat{f}(x, p)
$$

$$
\begin{aligned}
\left(\frac{\partial}{\partial t}+\frac{p}{\mu} \frac{\partial}{\partial x}\right)[|2\rangle\langle 1| \widehat{f}(x, p)]= & |2\rangle\langle 1| \int d p^{\prime} J_{12}\left(x, p-p^{\prime}\right) \widehat{f}\left(x, p^{\prime}\right)+ \\
& +i \Omega \exp \left(i \omega_{L} t\right)|2\rangle\langle 2| \widehat{f}(x, p)- \\
& -i \Omega \exp \left(i \omega_{L} t\right)|1\rangle\langle 1| \widehat{f}(x, p)+ \\
& +\frac{i \varkappa}{2}|3\rangle\langle 1| \widehat{f}(x, p)
\end{aligned}
$$

$$
\begin{aligned}
\left(\frac{\partial}{\partial t}+\frac{p}{\mu} \frac{\partial}{\partial x}\right)[|3\rangle\langle 2| \widehat{f}(x, p)]= & |3\rangle\langle 2| \int d p^{\prime} J_{32}\left(x, p-p^{\prime}\right) \widehat{f}\left(x, p^{\prime}\right)+ \\
& +i \Omega \exp \left(-i \omega_{L} t\right)|3\rangle\langle 2| \widehat{f}(x, p)+ \\
& +\frac{i \varkappa}{2}[|2\rangle\langle 2|-| 3\rangle\langle 3|] \widehat{f}(x, p)
\end{aligned}
$$

$$
\begin{aligned}
\left(\frac{\partial}{\partial t}+\frac{p}{\mu} \frac{\partial}{\partial x}\right)[|2\rangle\langle 3| \widehat{f}(x, p)]= & |2\rangle\langle 3| \int d p^{\prime} J_{23}\left(x, p^{\prime}-p\right) \widehat{f}\left(x, p^{\prime}\right)- \\
& -i \Omega \exp \left(i \omega_{L} t\right)|1\rangle\langle 3| \widehat{f}(x, p)+ \\
& +\frac{i \varkappa}{2}[|3\rangle\langle 3|-| 2\rangle\langle 2|] \widehat{f}(x, p)
\end{aligned}
$$

Novamente, no resultado das Equações (7.66) e (7.67), aparecem os operadores $|1\rangle\langle 3| \widehat{f}(x, p)$ e $|3\rangle\langle 1| \widehat{f}(x, p)$, o que nos leva a calcular as duas últimas equações de maneira a obter um sistema de equações diferenciais acopladas:

$$
\left(\frac{\partial}{\partial t}+\frac{p}{\mu} \frac{\partial}{\partial x}\right)[|1\rangle\langle 3| \widehat{f}(x, p)]=|1\rangle\langle 3| \int d p^{\prime} J_{13}\left(x, p^{\prime}-p\right) \widehat{f}\left(x, p^{\prime}\right)+
$$




$$
\begin{aligned}
& +i \Omega \exp \left(-i \omega_{L} t\right)|2\rangle\langle 3| \widehat{f}(x, p)- \\
& -\frac{i \varkappa}{2}|1\rangle\langle 2| \widehat{f}(x, p)
\end{aligned}
$$

$$
\begin{aligned}
\left(\frac{\partial}{\partial t}+\frac{p}{\mu} \frac{\partial}{\partial x}\right)[|3\rangle\langle 1| \widehat{f}(x, p)]= & |3\rangle\langle 1| \int d p^{\prime} J_{31}\left(x, p-p^{\prime}\right) \widehat{f}\left(x, p^{\prime}\right)+ \\
& +i \Omega \exp \left(i \omega_{L} t\right)|3\rangle\langle 2| \widehat{f}(x, p)+ \\
& +\frac{i \varkappa}{2}|2\rangle\langle 1| \widehat{f}(x, p) .
\end{aligned}
$$

A função $J_{m n}(x, p)$ ainda pode ser escrita como

$$
J_{m n}(x, p)=\frac{\sqrt{2 \pi \hbar}}{(i \hbar) \pi \hbar}\left[U_{m}(2 p) \exp \left(\frac{2 i p x}{\hbar}\right)-U_{n}(-2 p) \exp \left(-\frac{2 i p x}{\hbar}\right)\right]
$$

sendo

$$
U_{j}(k)=\frac{1}{\sqrt{2 \pi \hbar}} \int d x V_{j}(x) \exp \left(-\frac{i k x}{\hbar}\right)
$$

Expandindo (7.72) em série de potências e efetuando as integrações em $x$ e $p^{\prime}$ teremos

$$
\begin{gathered}
\int d p^{\prime} \rho_{m n}\left(x, p^{\prime}, t\right) J_{m n}\left(x, p-p^{\prime}\right)= \\
=\sum_{k=0}^{\infty} \frac{(\hbar i)^{k-1}}{2^{k} k !}\left[\frac{d^{k} V_{m}(x)}{d x^{k}}-(-1)^{k} \frac{d^{k} V_{n}(x)}{d x^{k}}\right] \frac{\partial^{k} \rho_{m n}(x, p, t)}{\partial p^{k}} .
\end{gathered}
$$

Conseguimos, desta maneira, uma série de potências de $\hbar$ para $\int d p^{\prime} \rho_{m n}\left(x, p^{\prime}, t\right)$ $J_{m n}\left(x, p-p^{\prime}\right)$. Assim, tomando os termos referentes a $k=0$ e $k=1$ temos

$$
\begin{aligned}
& \int d p^{\prime} \rho_{m n}\left(x, p^{\prime}, t\right) J_{m n}\left(x, p-p^{\prime}\right) \approx \\
\approx & (\hbar i)^{-1}\left[V_{m}(x)-V_{n}(x)\right] \rho_{m n}(x, p, t)
\end{aligned}
$$




$$
+\frac{1}{2}\left[\frac{d V_{m}(x)}{d x}+\frac{d V_{n}(x)}{d x}\right] \frac{\partial \rho_{m n}(x, p, t)}{\partial p}
$$

Definindo, ainda, $V_{g}(x)=V_{1}, V_{e}(x)=V_{2}-\hbar \omega_{0}$ e $V_{i}(x)=V_{3}-\hbar \omega_{0}+\hbar \varkappa$, onde $\hbar \omega_{0}$ é a diferença de energia entre os estados atômicos de simetrias ${ }^{2} P_{3 / 2}$ e ${ }^{2} S_{1 / 2}$ e $\hbar \varkappa$ é a separação entre ${ }^{2} P_{1 / 2} \mathrm{e}^{2} P_{3 / 2}$. As equações de (7.62) a (7.71) possuem os seguinte valores esperados:

$$
\begin{aligned}
& \left(\frac{\partial}{\partial t}+\frac{p}{\mu} \frac{\partial}{\partial x}\right)\langle\widehat{f}(x, p)\rangle=\frac{\partial}{\partial p}\left[V_{g}^{\prime}(x)\left\langle\widehat{s s^{\dagger}} \widehat{f}(x, p)\right\rangle+\right. \\
& +V_{e}^{\prime}(x)\left\langle\widehat{s}^{\dagger} \widehat{s} \widehat{f}(x, p)\right\rangle+ \\
& \left.+V_{i}^{\prime}(x)\left\langle\widehat{\sigma}^{\dagger} \widehat{\sigma} \widehat{f}(x, p)\right\rangle\right] \\
& \left(\frac{\partial}{\partial t}+\frac{p}{\mu} \frac{\partial}{\partial x}\right)\left\langle\widehat{s}^{\dagger} \widehat{f}(x, p)\right\rangle=\frac{\partial}{\partial p} V_{g}^{\prime}(x)\left\langle\widehat{s} \widehat{s}^{\dagger} \widehat{f}(x, p)\right\rangle+ \\
& -i \Omega\left\langle\exp \left(-i \omega_{L} t\right) \widehat{s}^{\dagger} \widehat{f}(x, p)\right\rangle+ \\
& +i \Omega\left\langle\exp \left(i \omega_{L} t\right) \widehat{s} \widehat{f}(x, p)\right\rangle \\
& \left(\frac{\partial}{\partial t}+\frac{p}{\mu} \frac{\partial}{\partial x}\right)\left\langle\widehat{s}^{\dagger} \widehat{s} \widehat{f}(x, p)\right\rangle=\frac{\partial}{\partial p} V_{e}^{\prime}(x)\left\langle\widehat{s}^{\dagger} \widehat{s} \widehat{f}(x, p)\right\rangle+ \\
& +i \Omega\left\langle\exp \left(-i \omega_{L} t\right) \hat{s}^{\dagger} \widehat{f}(x, p)\right\rangle \\
& -i \Omega\left\langle\exp \left(i \omega_{L} t\right) \hat{s} \widehat{f}(x, p)\right\rangle+ \\
& +\frac{i \varkappa}{2}\left\langle\left(\hat{\sigma}^{\dagger}-\widehat{\sigma}\right) \hat{f}(x, p)\right\rangle \\
& \left(\frac{\partial}{\partial t}+\frac{p}{\mu} \frac{\partial}{\partial x}\right)\left\langle\widehat{\sigma}^{\dagger} \widehat{\sigma} \widehat{f}(x, p)\right\rangle=\frac{\partial}{\partial p} V_{i}^{\prime}(x)\left\langle\widehat{\sigma}^{\dagger} \widehat{\sigma} \widehat{f}(x, p)\right\rangle+
\end{aligned}
$$




$$
\begin{aligned}
& \frac{i \varkappa}{2}\left\langle\left(\widehat{\sigma}-\widehat{\sigma}^{\dagger}\right) \widehat{f}(x, p)\right\rangle \\
& \left(\frac{\partial}{\partial t}+\frac{p}{\mu} \frac{\partial}{\partial x}\right)\left\langle\exp \left(i \omega_{L} t\right) \widehat{s} \widehat{f}(x, p)\right\rangle=\frac{1}{2} \frac{\partial}{\partial p}\left[V_{e}^{\prime}(x)+V_{g}^{\prime}(x)\right] \\
& \left\langle\exp \left(i \omega_{L} t\right) \widehat{s} \widehat{f}(x, p)\right\rangle+ \\
& +i\left[\Delta-\frac{V_{e}(x)-V_{g}(x)}{\hbar}\right] \\
& \left\langle\exp \left(i \omega_{L} t\right) \widehat{s} \widehat{f}(x, p)\right\rangle- \\
& -\frac{i \varkappa}{2}\left\langle\exp \left(i \omega_{L} t\right) \widehat{s} \widehat{\sigma} \widehat{f}(x, p)\right\rangle+ \\
& +i \Omega\left\langle\left(\widehat{s} \widehat{s}^{\dagger}-\widehat{s}^{\dagger} \widehat{s}\right) \widehat{f}(x, p)\right\rangle \\
& \left(\frac{\partial}{\partial t}+\frac{p}{\mu} \frac{\partial}{\partial x}\right)\left\langle\exp \left(-i \omega_{L} t\right) \widehat{s}^{\dagger} \widehat{f}(x, p)\right\rangle=\frac{1}{2} \frac{\partial}{\partial p}\left[V_{e}^{\prime}(x)+V_{g}^{\prime}(x)\right] \\
& \left\langle\exp \left(-i \omega_{L} t\right) \hat{s}^{\dagger} \hat{f}(x, p)\right\rangle- \\
& -i\left[\Delta+\frac{V_{e}(x)-V_{g}(x)}{\hbar}\right] \\
& \left\langle\exp \left(-i \omega_{L} t\right) \widehat{s}^{\dagger} \hat{f}(x, p)\right\rangle+ \\
& +\frac{i \varkappa}{2}\left\langle\exp \left(-i \omega_{L} t\right) \widehat{\sigma}^{\dagger} \widehat{s}^{\dagger} \widehat{f}(x, p)\right\rangle+ \\
& -i \Omega\left\langle\left(\widehat{s}^{\dagger} \widehat{s}-\widehat{s}^{\dagger}\right) \widehat{f}(x, p)\right\rangle, \\
& \left(\frac{\partial}{\partial t}+\frac{p}{\mu} \frac{\partial}{\partial x}\right)\left\langle\hat{\sigma}^{\dagger} \widehat{f}(x, p)\right\rangle=\frac{1}{2} \frac{\partial}{\partial p}\left[V_{e}^{\prime}(x)+V_{i}^{\prime}(x)\right]\left\langle\widehat{\sigma}^{\dagger} \widehat{f}(x, p)\right\rangle+ \\
& -i\left[\varkappa-\frac{V_{i}(x)-V_{e}(x)}{\hbar}\right]\left\langle\widehat{\sigma}^{\dagger} \widehat{f}(x, p)\right\rangle+ \\
& +\frac{i \varkappa}{2}\left\langle\left(\widehat{\sigma}^{\dagger} \widehat{\sigma}-\widehat{\sigma} \widehat{\sigma}^{\dagger}\right) \widehat{f}(x, p)\right\rangle+
\end{aligned}
$$




$$
+i \Omega\left\langle\widehat{\sigma}^{\dagger} \widehat{s}^{\dagger} \exp \left(-i \omega_{L} t\right) \widehat{f}(x, p)\right\rangle
$$

$$
\begin{aligned}
\left(\frac{\partial}{\partial t}+\frac{p}{\mu} \frac{\partial}{\partial x}\right)\langle\widehat{\sigma} \widehat{f}(x, p)\rangle= & \frac{1}{2} \frac{\partial}{\partial p}\left[V_{e}^{\prime}(x)+V_{i}^{\prime}(x)\right]\langle\widehat{\sigma} \widehat{f}(x, p)\rangle+ \\
& +i\left[\varkappa+\frac{V_{e}(x)-V_{i}(x)}{\hbar}\right]\langle\widehat{\sigma} \widehat{f}(x, p)\rangle+ \\
& +\frac{i \varkappa}{2}\left\langle\left(\widehat{s}^{\dagger} \widehat{s}-\widehat{\sigma}^{\dagger} \widehat{\sigma}\right) \widehat{f}(x, p)\right\rangle- \\
& -i \Omega\left\langle\widehat{s} \widehat{\sigma} \exp \left(i \omega_{L} t\right) \widehat{f}(x, p)\right\rangle
\end{aligned}
$$

$$
\begin{aligned}
\left(\frac{\partial}{\partial t}+\frac{p}{\mu} \frac{\partial}{\partial x}\right)\left\langle\exp \left(i \omega_{L} t\right) \widehat{s} \widehat{\sigma} \widehat{f}(x, p)\right\rangle= & \frac{1}{2} \frac{\partial}{\partial p}\left[V_{g}^{\prime}(x)+V_{i}^{\prime}(x)\right] \\
& \left\langle\exp \left(i \omega_{L} t\right) \widehat{s} \widehat{\sigma} \widehat{f}(x, p)\right\rangle+ \\
& +i\left[\Delta+\varkappa+\frac{V_{g}(x)-V_{i}(x)}{\hbar}\right] \\
& \left\langle\exp \left(i \omega_{L} t\right) \widehat{s} \widehat{\sigma} \widehat{f}(x, p)\right\rangle+ \\
& -\frac{i \varkappa}{2}\langle\exp \widehat{s} \widehat{f}(x, p)\rangle \\
& -i \Omega\langle\widehat{\sigma} \widehat{f}(x, p)\rangle
\end{aligned}
$$

$$
\begin{aligned}
\left(\frac{\partial}{\partial t}+\frac{p}{\mu} \frac{\partial}{\partial x}\right)\left\langle\exp \left(-i \omega_{L} t\right) \widehat{\sigma}^{\dagger} \widehat{s}^{\dagger} \widehat{f}(x, p)\right\rangle= & \frac{1}{2} \frac{\partial}{\partial p}\left[V_{g}^{\prime}(x)+V_{i}^{\prime}(x)\right] \\
& \left\langle\exp \left(-i \omega_{L} t\right) \widehat{\sigma}^{\dagger} \widehat{s}^{\dagger} \widehat{f}(x, p)\right\rangle+ \\
& -i\left[\Delta+\varkappa+\frac{V_{g}(x)-V_{i}(x)}{\hbar}\right] \\
& \left\langle\exp \left(-i \omega_{L} t\right) \widehat{\sigma}^{\dagger} \widehat{s}^{\dagger} \widehat{f}(x, p)\right\rangle+ \\
& -\frac{i \varkappa}{2}\left\langle\exp \left(-i \omega_{L} t\right) \widehat{s}^{\dagger} \widehat{f}(x, p)\right\rangle+
\end{aligned}
$$




$$
+i \Omega\left\langle\widehat{\sigma}^{\dagger} \widehat{f}(x, p)\right\rangle
$$

onde $V_{g}(x)=V_{1}(x), V_{e}(x)=V_{2}(x)-\hbar \omega_{0}$ e $V_{i}(x)=V_{3}(x)-\hbar \omega_{0}+\hbar \varkappa$ e $\Delta=\omega_{L}-\omega_{0}$. Chamando os valores esperados do operador de Wigner de $\rho_{11}(x, p, t)=\left\langle\widehat{s} \widehat{s}^{\dagger} \widehat{f}(x, p)\right\rangle$, $\rho_{22}(x, p, t)=\left\langle\widehat{s}^{\dagger} \widehat{s} \widehat{f}(x, p)\right\rangle, \rho_{33}(x, p, t)=\left\langle\widehat{\sigma}^{\dagger} \widehat{\sigma} \widehat{f}(x, p)\right\rangle, \rho_{12}(x, p, t)=$ $\left\langle\exp \left(-i \omega_{L} t\right) \widehat{s}^{\dagger} \widehat{f}(x, p)\right\rangle, \rho_{13}(x, p, t)=\left\langle\exp \left(-i \omega_{L} t\right) \widehat{\sigma}^{\dagger} \widehat{s}^{\dagger} \widehat{f}(x, p)\right\rangle, \rho_{23}(x, p, t)=\left\langle\widehat{\sigma}^{\dagger} \widehat{f}(x, p)\right\rangle$, e seus respectivos complexo conjugados $\rho_{21}(x, p, t)=\rho_{12}^{*}(x, p, t), \rho_{31}(x, p, t)=\rho_{13}^{*}(x, p, t)$ e $\rho_{32}(x, p, t)=\rho_{23}^{*}(x, p, t)$ obtemos

$$
\begin{aligned}
\left(\frac{\partial}{\partial t}+\frac{p}{\mu} \frac{\partial}{\partial x}\right) \rho_{11}(x, p, t)= & V_{g}^{\prime}(x) \frac{\partial}{\partial p} \rho_{11}(x, p, t)+ \\
& +i \Omega\left[-\rho_{12}(x, p, t)+\rho_{21}(x, p, t)\right]
\end{aligned}
$$

$$
\begin{aligned}
\left(\frac{\partial}{\partial t}+\frac{p}{\mu} \frac{\partial}{\partial x}\right) \rho_{22}(x, p, t)= & V_{e}^{\prime}(x) \frac{\partial}{\partial p} \rho_{22}(x, p, t)+ \\
& +i \Omega\left[\rho_{12}(x, p, t)-\rho_{21}(x, p, t)\right]+ \\
& +\frac{i \varkappa}{2}\left[\rho_{23}(x, p, t)-\rho_{32}(x, p, t)\right]
\end{aligned}
$$

$$
\begin{aligned}
\left(\frac{\partial}{\partial t}+\frac{p}{\mu} \frac{\partial}{\partial x}\right) \rho_{33}(x, p, t)= & V_{i}^{\prime}(x) \frac{\partial}{\partial p} \rho_{33}(x, p, t)+ \\
& +\frac{i \varkappa}{2}\left[\rho_{32}(x, p, t)-\rho_{23}(x, p, t)\right]
\end{aligned}
$$

$$
\left(\frac{\partial}{\partial t}+\frac{p}{\mu} \frac{\partial}{\partial x}\right) \rho_{21}(x, p, t)=\frac{1}{2}\left[V_{e}^{\prime}(x)+V_{g}^{\prime}(x)\right] \frac{\partial}{\partial p} \rho_{21}(x, p, t)+
$$




$$
\begin{aligned}
& +i\left[\Delta-\frac{V_{e}(x)-V_{g}(x)}{\hbar}\right] \rho_{21}(x, p, t)+ \\
& +i \Omega\left[\rho_{11}(x, p, t)-\rho_{22}(x, p, t)\right]- \\
& -\frac{i \varkappa}{2} \rho_{31}(x, p, t)
\end{aligned}
$$

$$
\begin{aligned}
\left(\frac{\partial}{\partial t}+\frac{p}{\mu} \frac{\partial}{\partial x}\right) \rho_{12}(x, p, t)= & \frac{1}{2}\left[V_{e}^{\prime}(x)+V_{g}^{\prime}(x)\right] \frac{\partial}{\partial p} \rho_{12}(x, p, t)+ \\
& -i\left[\Delta-\frac{V_{e}(x)-V_{g}(x)}{\hbar}\right] \rho_{12}(x, p, t)+ \\
& +i \Omega\left[\rho_{22}(x, p, t)-\rho_{11}(x, p, t)\right]+ \\
& +\frac{i \varkappa}{2} \rho_{13}(x, p, t)
\end{aligned}
$$

$$
\begin{aligned}
\left(\frac{\partial}{\partial t}+\frac{p}{\mu} \frac{\partial}{\partial x}\right) \rho_{23}(x, p, t)= & \frac{1}{2}\left[V_{e}^{\prime}(x)+V_{i}^{\prime}(x)\right] \frac{\partial}{\partial p} \rho_{23}(x, p, t)+ \\
& -i\left[\varkappa-\frac{V_{i}(x)-V_{e}(x)}{\hbar}\right] \rho_{23}(x, p, t)+ \\
& +\frac{i \varkappa}{2}\left[\rho_{33}(x, p, t)-\rho_{22}(x, p, t)\right]+ \\
& +i \Omega \rho_{13}(x, p, t),
\end{aligned}
$$

$$
\begin{aligned}
\left(\frac{\partial}{\partial t}+\frac{p}{\mu} \frac{\partial}{\partial x}\right) \rho_{32}(x, p, t)= & \frac{1}{2}\left[V_{e}^{\prime}(x)+V_{i}^{\prime}(x)\right] \frac{\partial}{\partial p} \rho_{32}(x, p, t)+ \\
& +i\left[\varkappa+\frac{V_{e}(x)-V_{i}(x)}{\hbar}\right] \rho_{32}(x, p, t)+ \\
& +\frac{i \varkappa}{2}\left[\rho_{22}(x, p, t)-\rho_{33}(x, p, t)\right]- \\
& -i \Omega \rho_{31}(x, p, t),
\end{aligned}
$$




$$
\begin{aligned}
\left(\frac{\partial}{\partial t}+\frac{p}{\mu} \frac{\partial}{\partial x}\right) \rho_{31}(x, p, t)= & \frac{1}{2}\left[V_{g}^{\prime}(x)+V_{i}^{\prime}(x)\right] \frac{\partial}{\partial p} \rho_{31}(x, p, t)+ \\
& +i\left[\Delta+\varkappa+\frac{V_{g}(x)-V_{i}(x)}{\hbar}\right] \rho_{31}(x, p, t)+ \\
& -\frac{i \varkappa}{2} \rho_{21}(x, p, t)+i \Omega \rho_{32}(x, p, t), \\
\left(\frac{\partial}{\partial t}+\frac{p}{\mu} \frac{\partial}{\partial x}\right) \rho_{13}(x, p, t)= & \frac{1}{2}\left[V_{g}^{\prime}(x)+V_{i}^{\prime}(x)\right] \frac{\partial}{\partial p} \rho_{13}(x, p, t)+ \\
& -i\left[\Delta+\varkappa+\frac{V_{g}(x)-V_{i}(x)}{\hbar}\right] \rho_{13}(x, p, t)+ \\
& -\frac{i \varkappa}{2} \rho_{12}(x, p, t)+i \Omega \rho_{23}(x, p, t) .
\end{aligned}
$$




\section{Apêndice C: Soluções Analíticas}

Com a separação da equação

$$
\frac{\partial \hat{U}}{\partial t}=\left(\hat{M}_{12}+\hat{M}_{23}\right) \hat{U}
$$

em

$$
\begin{aligned}
& \frac{\partial \hat{U}}{\partial t}=\hat{M}_{12} \hat{U} \\
& \frac{\partial \hat{U}}{\partial t}=\hat{M}_{23} \hat{U}
\end{aligned}
$$

pelo uso do operador partido, chegamos a duas equações diferenciais que possuem soluções analíticas, uma vez que

$$
\hat{M}_{12}=\left(\begin{array}{ccccccccc}
0 & -i \Omega & 0 & i \Omega & 0 & 0 & 0 & 0 & 0 \\
-i \Omega & -\frac{i \Delta_{12}}{2} & 0 & 0 & i \Omega & 0 & 0 & 0 & 0 \\
0 & 0 & \frac{i \Delta_{13}}{2} & 0 & 0 & i \Omega & 0 & 0 & 0 \\
i \Omega & 0 & 0 & \frac{i \Delta_{12}}{2} & -i \Omega & 0 & 0 & 0 & 0 \\
0 & i \Omega & 0 & -i \Omega & 0 & 0 & 0 & 0 & 0 \\
0 & 0 & i \Omega & 0 & 0 & \frac{i \Delta_{23}}{2} & 0 & 0 & 0 \\
0 & 0 & 0 & 0 & 0 & 0 & -\frac{i \Delta_{13}}{2} & -i \Omega & 0 \\
0 & 0 & 0 & 0 & 0 & 0 & -i \Omega & -\frac{i \Delta_{23}}{2} & 0 \\
0 & 0 & 0 & 0 & 0 & 0 & 0 & 0 & 0
\end{array}\right)
$$

e

$$
\hat{M}_{23}=\left(\begin{array}{ccccccccc}
0 & 0 & 0 & 0 & 0 & 0 & 0 & 0 & 0 \\
0 & -\frac{i \Delta_{12}}{2} & i \frac{\chi}{2} & 0 & 0 & 0 & 0 & 0 & 0 \\
0 & i \frac{\chi}{2} & \frac{i \Delta_{13}}{2} & 0 & 0 & 0 & 0 & 0 & 0 \\
0 & 0 & 0 & \frac{i \Delta_{12}}{2} & 0 & 0 & -i \frac{\chi}{2} & 0 & 0 \\
0 & 0 & 0 & 0 & 0 & i \frac{\chi}{2} & 0 & -i \frac{\chi}{2} & 0 \\
0 & 0 & 0 & 0 & i \frac{\chi}{2} & \frac{i \Delta_{23}}{2} & 0 & 0 & -i \frac{\chi}{2} \\
0 & 0 & 0 & -i \frac{\chi}{2} & 0 & 0 & -\frac{i \Delta_{13}}{2} & 0 & 0 \\
0 & 0 & 0 & 0 & -i \frac{\chi}{2} & 0 & 0 & -\frac{i \Delta_{23}}{2} & i \frac{\chi}{2} \\
0 & 0 & 0 & 0 & 0 & -i \frac{\chi}{2} & 0 & i \frac{\chi}{2} & 0
\end{array}\right)
$$


Após algumas manipulações simples, chegamos à solução da equação (8.93), que é apresentada a seguir.

$$
\begin{aligned}
& \rho_{11}(x, p, t)=\rho_{11}(x, p, 0)+\frac{\Omega}{\omega_{12}} \operatorname{Im}\left[\rho_{12}(x, p, 0)\right] \sin \left(2 \omega_{12} t\right)+ \\
& -\frac{\Omega}{\omega_{12}}\left\{\frac{\Delta_{12}}{4 \omega_{12}} \operatorname{Re}\left[\rho_{12}(x, p, 0)\right]+\right. \\
& \left.-\frac{\Omega}{2 \omega_{12}} \operatorname{Re}\left[\rho_{11}(x, p, 0)\right]-\operatorname{Re}\left[\rho_{22}(x, p, 0)\right]\right\}\left[\cos \left(2 \omega_{12} t\right)-1\right], \\
& \rho_{12}(x, p, t)=\operatorname{Re}\left[\rho_{12}(x, p, 0)\right]-\frac{\Delta_{12}}{4 \omega_{12}} \operatorname{Im}\left[\rho_{12}(x, p, 0)\right] \sin \left(2 \omega_{12} t\right)+ \\
& +\frac{\Delta_{12}}{4 \omega_{12}}\left\{\frac{\Delta_{12}}{4 \omega_{12}} \operatorname{Re}\left[\rho_{12}(x, p, 0)\right]+\right. \\
& \left.-\frac{\Omega}{2 \omega_{12}} \operatorname{Re}\left[\rho_{11}(x, p, 0)\right]-\operatorname{Re}\left[\rho_{22}(x, p, 0)\right]\right\}\left[\cos \left(2 \omega_{12} t\right)-1\right]+ \\
& +i\left\{\operatorname{Im}\left[\rho_{12}(x, p, 0)\right] \cos \left(2 \omega_{12} t\right)+\right. \\
& +\left[\frac{\Delta_{12}}{4 \omega_{12}} \operatorname{Re}\left[\rho_{12}(x, p, 0)\right]-\frac{\Omega}{2 \omega_{12}} \operatorname{Re}\left[\rho_{11}(x, p, 0)\right]+\right. \\
& \left.\left.-\operatorname{Re}\left[\rho_{22}(x, p, 0)\right]\right] \sin \left(2 \omega_{12} t\right)\right\} \text {, } \\
& \rho_{13}(x, p, t)=\left\{\frac{\left\{\left[\left(\frac{\Delta_{13}+\Delta_{23}}{4}\right)+\frac{\lambda_{12}}{2}\right]-\frac{\Delta_{13}}{2}\right\} \rho_{13}(x, p, 0)}{\lambda_{12}}+\right. \\
& \left.-\frac{\Omega \rho_{23}(x, p, 0)}{\lambda_{12}}\right\} \exp \left\{i\left[\left(\frac{\Delta_{13}+\Delta_{23}}{4}\right)-\frac{\lambda_{12}}{2}\right] t\right\}+ \\
& +\left\{\frac{\left\{\frac{\Delta_{13}}{2}-\left[\left(\frac{\Delta_{13}+\Delta_{23}}{4}\right)-\frac{\lambda_{12}}{2}\right]\right\} \rho_{13}(x, p, 0)}{\lambda_{12}}+\right. \\
& \left.+\frac{\Omega \rho_{23}(x, p, 0)}{\lambda_{12}}\right\} \exp \left\{i\left[\left(\frac{\Delta_{13}+\Delta_{23}}{4}\right)-\frac{\lambda_{12}}{2}\right] t\right\} \text {, } \\
& \rho_{21}(x, p, t)=\rho_{12}^{*}(x, p, t),
\end{aligned}
$$




$$
\begin{aligned}
& \rho_{22}(x, p, t)=\rho_{22}(x, p, 0)-\frac{\Omega}{\omega_{12}} \operatorname{Im}\left[\rho_{12}(x, p, 0)\right] \sin \left(2 \omega_{12} t\right)+ \\
& +\frac{\Omega}{\omega_{12}}\left\{\frac{\Delta_{12}}{4 \omega_{12}} \operatorname{Re}\left[\rho_{12}(x, p, 0)\right]-\frac{\Omega}{2 \omega_{12}} \operatorname{Re}\left[\rho_{11}(x, p, 0)\right]+\right. \\
& \left.-\operatorname{Re}\left[\rho_{22}(x, p, 0)\right]\right\}\left[\cos \left(2 \omega_{12} t\right)-1\right] \text {, } \\
& \rho_{23}(x, p, t)=\left\{\frac{\left\{i\left[\left(\frac{\Delta_{13}+\Delta_{23}}{4}\right)+\frac{\lambda_{12}}{2}\right]-i \frac{\Delta_{13}}{2}\right\} \rho_{13}(x, p, 0)}{\lambda_{12}}+\right. \\
& \left.-\frac{i \Omega \rho_{23}(x, p, 0)}{\lambda_{12}}\right\}\left[\frac{\Delta_{13}}{2}-\left(\frac{\Delta_{13}+\Delta_{23}}{4}\right)-\frac{1}{2} \lambda_{12}\right] \\
& \frac{i}{\Omega} \exp \left\{i\left[\left(\frac{\Delta_{13}+\Delta_{23}}{4}\right)-\frac{\lambda_{12}}{2}\right] t\right\}+ \\
& +\left\{\frac{\left\{i \frac{\Delta_{13}}{2}-i\left[\left(\frac{\Delta_{13}+\Delta_{23}}{4}\right)-\frac{\lambda_{12}}{2}\right]\right\} \rho_{13}(x, p, 0)}{\lambda_{12}}+\right. \\
& \left.+\frac{i \Omega \rho_{23}(x, p, 0)}{\lambda_{12}}\right\}\left\{\frac{\Delta_{13}}{2}-\left[\left(\frac{\Delta_{13}+\Delta_{23}}{4}\right)+\frac{\lambda_{12}}{2}\right]\right\} \\
& \frac{i}{\Omega} \exp \left\{i\left[\left(\frac{\Delta_{13}+\Delta_{23}}{4}\right)+\frac{\lambda_{12}}{2}\right] t\right\} \\
& \rho_{31}(x, p, t)=\rho_{31}^{*}(x, p, t) \\
& \rho_{32}(x, p, t)=\rho_{32}^{*}(x, p, t) \\
& \rho_{33}(x, p, t)=\rho_{33}(x, p, 0)
\end{aligned}
$$

O valor de $\omega_{12}$ é dado por

$$
\lambda_{12}=\sqrt{\left(\frac{\Delta_{13}+\Delta_{23}}{2}\right)^{2}+4 \Omega^{2}}
$$




$$
\omega_{12}=\sqrt{\left(\frac{\Delta_{12}}{4}\right)^{2}+\Omega^{2}} .
$$

Da mesma forma obtivemos as soluções analíticas para (8.94)

$$
\begin{aligned}
& \rho_{11}(x, p, t)=\rho_{11}(x, p, 0), \\
& \rho_{12}(x, p, t)=\frac{\left\{\left[\left(\frac{\Delta_{12}+\Delta_{13}}{4}\right)+\frac{\lambda_{23}}{2}\right]-\frac{\Delta_{12}}{2}\right\} \rho_{12}(x, p, 0)-\frac{\chi}{2} \rho_{13}(x, p, 0)}{\lambda_{23}} \\
& \exp \left\{i\left[\left(\frac{\Delta_{12}+\Delta_{13}}{4}\right)-\frac{\lambda_{23}}{2}\right] t\right\}+ \\
& +\frac{\left\{\frac{\Delta_{12}}{2}-\left[\left(\frac{\Delta_{12}+\Delta_{13}}{4}\right)-\frac{\lambda_{23}}{2}\right]\right\} \rho_{12}(x, p, 0)+\frac{\chi}{2} \rho_{13}(x, p, 0)}{\lambda_{23}} \\
& \exp \left\{i\left[\left(\frac{\Delta_{12}+\Delta_{13}}{4}\right)+\frac{\lambda_{23}}{2}\right] t\right\}, \\
& \rho_{13}(x, p, t)=\frac{\left\{i\left[\left(\frac{\Delta_{12}+\Delta_{13}}{4}\right)+\frac{\lambda_{23}}{2}\right]-i \frac{\Delta_{12}}{2}\right\} \rho_{12}(x, p, 0)-i \frac{\chi}{2} \rho_{13}(x, p, 0)}{\lambda_{23}} \\
& \left\{\frac{\Delta_{12}}{2}-\left[\left(\frac{\Delta_{12}+\Delta_{13}}{4}\right)-\frac{\lambda_{23}}{2}\right]\right\} \\
& \frac{2 i}{\chi} \exp \left\{i\left[\left(\frac{\Delta_{12}+\Delta_{13}}{4}\right)-\frac{\lambda_{23}}{2}\right] t\right\}+ \\
& +\frac{\left\{i \frac{\Delta_{12}}{2}-i\left[\left(\frac{\Delta_{12}+\Delta_{13}}{4}\right)-\frac{\lambda_{23}}{2}\right]\right\} \rho_{12}(x, p, 0)+i \frac{\chi}{2} \rho_{13}(x, p, 0)}{\lambda_{23}} \\
& \left\{\frac{\Delta_{12}}{2}-\left[\left(\frac{\Delta_{12}+\Delta_{13}}{4}\right)+\frac{\lambda_{23}}{2}\right]\right\} \\
& \frac{2 i}{\chi} \exp \left\{i\left[\left(\frac{\Delta_{12}+\Delta_{13}}{4}\right)+\frac{\lambda_{23}}{2}\right] t\right\} \text {, } \\
& \rho_{21}(x, p, t)=\rho_{12}^{*}(x, p, t),
\end{aligned}
$$




$$
\begin{aligned}
& \rho_{22}(x, p, t)=\rho_{22}(x, p, 0)-\frac{\chi}{2 \omega_{23}} \operatorname{Im}\left[\rho_{23}(x, p, 0)\right] \sin \left(2 \omega_{23} t\right)+ \\
& +\frac{\chi}{2 \omega_{23}}\left\{\frac{\Delta_{23}}{4 \omega_{23}} \operatorname{Re}\left[\rho_{23}(x, p, 0)\right]+\right. \\
& \left.+\frac{\chi}{4 \omega_{23}}\left[\operatorname{Re}\left[\rho_{22}(x, p, 0)\right]\right]\right\}\left[\cos \left(2 \omega_{23} t\right)-1\right] \text {, } \\
& \rho_{23}(x, p, t)=\operatorname{Re}\left[\rho_{23}(x, p, 0)\right]-\frac{\Delta_{23}}{4 \omega_{23}} \operatorname{Im}\left[\rho_{23}(x, p, 0)\right] \sin \left(2 \omega_{23} t\right)+ \\
& +\frac{\Delta_{23}}{4 \omega_{23}}\left\{\frac{\Delta_{23}}{4 \omega_{23}} \operatorname{Re}\left[\rho_{23}(x, p, 0)\right]+\right. \\
& \left.+\frac{\chi}{4 \omega_{23}}\left[\operatorname{Re}\left[\rho_{22}(x, p, 0)\right]\right]\right\}\left[\cos \left(2 \omega_{23} t\right)-1\right]+ \\
& +i\left\{\operatorname{Im}\left[\rho_{23}(x, p, 0)\right] \cos \left(2 \omega_{23} t\right)+\left\{\frac{\Delta_{23}}{4 \omega_{23}} \operatorname{Re}\left[\rho_{23}(x, p, 0)\right]+\right.\right. \\
& \left.\left.+\frac{\chi}{4 \omega_{23}}\left[\operatorname{Re}\left[\rho_{22}(x, p, 0)\right]\right]\right\} \sin \left(2 \omega_{23} t\right)\right\} \\
& \rho_{31}(x, p, t)=\rho_{31}^{*}(x, p, t) \\
& \rho_{32}(x, p, t)=\rho_{32}^{\star}(x, p, t) \\
& \rho_{33}(x, p, t)=\rho_{33}(x, p, 0)+\frac{\chi}{2 \omega_{23}} \operatorname{Im}\left[\rho_{23}(x, p, 0)\right] \sin \left(2 \omega_{23} t\right)+ \\
& -\frac{\chi}{2 \omega_{23}}\left\{\frac{\Delta_{23}}{4 \omega_{23}} \operatorname{Re}\left[\rho_{23}(x, p, 0)\right]+\right. \\
& \left.+\frac{\chi}{4 \omega_{23}}\left[\operatorname{Re}\left[\rho_{22}(x, p, 0)\right]\right]\right\}\left[\cos \left(2 \omega_{23} t\right)-1\right] \text {, }
\end{aligned}
$$

onde

$$
\lambda_{23}=\sqrt{\left(\frac{\Delta_{12}}{2}-\frac{\Delta_{13}}{2}\right)^{2}+\left(\frac{4 \chi}{2}\right)^{2}},
$$




$$
\omega_{23}=\sqrt{\left(\frac{\Delta_{23}}{4}\right)^{2}+\Omega^{2}}
$$

Incluindo os termos de decaimento na Eq. (8.92) teremos ainda uma outra equação a solucionar:

$$
\frac{\partial \hat{U}}{\partial t}=\hat{M}_{21}^{\Gamma} \hat{U}
$$

onde

$$
\hat{M}_{21}^{\Gamma}=\left(\begin{array}{ccccccccc}
0 & 0 & 0 & 0 & \Gamma_{21} & 0 & 0 & 0 & 0 \\
0 & -\frac{\Gamma_{21}}{2} & 0 & 0 & 0 & 0 & 0 & 0 & 0 \\
0 & 0 & 0 & 0 & 0 & 0 & 0 & 0 & 0 \\
0 & 0 & 0 & -\frac{\Gamma_{21}}{2} & 0 & 0 & 0 & 0 & 0 \\
0 & 0 & 0 & 0 & -\Gamma_{21} & 0 & 0 & 0 & 0 \\
0 & 0 & 0 & 0 & 0 & 0 & 0 & 0 & 0 \\
0 & 0 & 0 & 0 & 0 & 0 & 0 & 0 & 0 \\
0 & 0 & 0 & 0 & 0 & 0 & 0 & 0 & 0 \\
0 & 0 & 0 & 0 & 0 & 0 & 0 & 0 & 0
\end{array}\right),
$$

que possui solução analítica simples:

$$
\begin{aligned}
& \rho_{11}(x, p, t)=\left[1-\exp \left(-\Gamma_{21} t\right)\right] \rho_{22}(x, p, 0)+\rho_{11}(x, p, 0) \\
& \rho_{12}(x, p, t)=\exp \left(-\frac{\Gamma_{21}}{2} t\right) \rho_{12}(x, p, 0) \\
& \rho_{13}(x, p, t)=\rho_{13}(x, p, 0) \\
& \rho_{21}(x, p, t)=\exp \left(-\frac{\Gamma_{21}}{2} t\right) \rho_{21}(x, p, 0) \\
& \rho_{22}(x, p, t)=\exp \left(-\Gamma_{21} t\right) \rho_{22}(x, p, 0) \\
& \rho_{23}(x, p, t)=\rho_{23}(x, p, 0) \\
& \rho_{31}(x, p, t)=\rho_{31}(x, p, 0) \\
& \rho_{32}(x, p, t)=\rho_{32}(x, p, 0)
\end{aligned}
$$




$$
\rho_{33}(x, p, t)=\rho_{33}(x, p, 0) .
$$

